\title{
Development of a new Human Identity Testing Kit for the Simultaneous Amplification of the 13 CODIS STR Loci plus Amelogenin Using Locus Specific Brackets (LSBs)
}

Lizmery Suarez Ferguson

West Virginia University

Follow this and additional works at: https://researchrepository.wvu.edu/etd

\section{Recommended Citation}

Suarez Ferguson, Lizmery, "Development of a new Human Identity Testing Kit for the Simultaneous Amplification of the 13 CODIS STR Loci plus Amelogenin Using Locus Specific Brackets (LSBs)" (2012). Graduate Theses, Dissertations, and Problem Reports. 4926.

https://researchrepository.wvu.edu/etd/4926

This Thesis is protected by copyright and/or related rights. It has been brought to you by the The Research Repository @ WVU with permission from the rights-holder(s). You are free to use this Thesis in any way that is permitted by the copyright and related rights legislation that applies to your use. For other uses you must obtain permission from the rights-holder(s) directly, unless additional rights are indicated by a Creative Commons license in the record and/ or on the work itself. This Thesis has been accepted for inclusion in WVU Graduate Theses, Dissertations, and Problem Reports collection by an authorized administrator of The Research Repository @ WVU. For more information, please contact researchrepository@mail.wvu.edu. 


\title{
Development of a new Human Identity Testing Kit for the Simultaneous Amplification of the 13 CODIS STR Loci plus Amelogenin Using Locus Specific Brackets (LSBs)
}

\section{Lizmery Suarez Ferguson}

\author{
Thesis \\ Submitted to the Eberly College of Arts and Sciences \\ at West Virginia University \\ in partial fulfillment of the requirements for the degree of \\ Masters of Science \\ in \\ Cellular and Molecular Biology
}

\section{Committee Members:}

Jeffrey Wells, Ph.D., Chair

Clifton Bishop. Ph.D.

Keith Morris Ph. D.

Department of Biology

Morgantown, West Virginia

2012

Key Words: Locus-Specific Brackets Multiplex. PCR, STRs, Forensic DNA 


\begin{abstract}
Development of a new Human Identity Testing Kit for the Simultaneous Amplification of the 13 CODIS STR Loci plus Amelogenin Using Locus Specific Brackets (LSBs)
\end{abstract}

\author{
Lizmery Suarez Ferguson
}

STR loci consist of short, repetitive sequence elements 2-7 base pairs in length. An STR genotype is typically determined by PCR amplification followed by fragment size estimation with electrophoresis. Commercial multiplex STR amplification kits are available for the 13 CORE loci used in the USA for human identity testing. The genotyping rationale with these multiplex STR amplification kits involves a two-fold calibration analysis: 1) An internal size standard (ISS) labeled with a separate dye establishes the size of the unknown DNA fragment and 2) an allelic ladder which contains most of the common alleles for each locus is also sized by the internal sized standard and used as a reference for the allele call. Locus-Specific Brackets (LSBs) offer an alternative STR sizing method that may be less susceptible to the problems caused by variation in electrophoretic conditions. An LSB multiplex may also be cheaper to produce and use than a conventional kit. LSBs are internal size standards that flank each targeted locus. The brackets have the same repeat structure as the locus, are 1 or more repeat units shorter or longer than the common alleles, and are labeled with the same dye. Because LSBs have the same electrophoretic properties as the sample alleles, their sizing function should not be impaired by injection-to-injection variation in conditions. The aim of this project was to produce an LSBbased human STR multiplex reaction kit, free of allelic dropout and with appropriate LSB size standards for analysis by custom genotyping software, and to do a simple performance evaluation of the kit by testing the reproducibility of profiles and the sensitivity of the reaction. The result was a new human STR multiplex that can amplify all 13 STR loci plus amelogenin in a single reaction. The new LSB multiplex has a sensitivity range of $0.1-1$ ng of DNA template where full genetic profiles can be produced. Also developed were new locus specific brackets needed to accommodate necessary shifts allele size range. Existing software failed to successfully genotype all alleles; however manual analysis of the data showed the correct genotypes for $81 \%$ of all tested alleles, with few incorrect calls as fragment were incorrectly sized by the size standard. Although the further development of the kit is clearly required, the LSB kit remains promising as a new and alternative method for forensic DNA analysis. 


\section{DEDICATION}

This thesis is dedicated to my mother who taught me that education is the way forward and hard work is the path to success 


\section{Acknowledgements}

I would first like to express my gratitude to Dr. Jeffrey Wells for giving me the opportunity to

obtain a master's degree under his supervision and whose expertise and understanding,

contributed in great manner to my research experience. I would also like to thank the members of my committee Dr. Clifton Bishop and Dr. Keith Morris for their support and advice throughout the development of this project. Special thanks go to a former lab member Dr. Christine Picard for helping me understand and develop the necessary skills needed to complete this project. I would also thank both a former and current member of our lab, Dr. Baneshwar Singh and Anne Perez (respectively) for their support and encouragement through the most challenging times of my graduate experience. In addition I will like to thank the faculty and graduate students of the WVU biology department whose support I greatly appreciate. Most importantly, I would like to thank God, my family, friends and loved ones who in many ways supported me and encouraged me to continue until the end. 


\section{Table of Contents}

$\begin{array}{ll}\text { Title page } & \text { i }\end{array}$

Abstract

Dedication $\quad$ iii

Acknowledgments iv

Table of Contents $\quad$ v

List of Tables $\quad$ vi

List of Figures $\quad$ vii

Part One: Development of a new Multiplex for the Simultaneous Amplification of the13 CODIS STR Loci plus Amelogenin using Locus Specific Brackets (LSBs)

Introduction

Materials \& Methods

Results

Part Two: Design and synthesis of Locus Specific Brackets

Introduction

Materials \& Methods

42-46

Results

Part Three: Preliminary evaluation of the Performance of Locus Specific Brackets as a new Human Identification Testing Kit

Introduction

Materials \& Methods

60-62

Results

63-65

Discussion \& Future Research

76-81

References

82-86

Appendix

89-95 


\section{List of Tables}

1. New primers for D13S317, vWA, D18S51 \& CSFIPO STR loci 21

2. Stutter percentage on various STR loci with the LSB multiplex 22

3. FAM/JOE-Bracket Mixtures concentrations $\quad 48$

4. FAM-labeled Locus Specific Brackets precision study (no sample present) 49

5. JOE-labeled Locus Specific Brackets precision Study $\quad 50$

6. Average peak Height Ratio after all three sample injections were averaged $\mathbf{6 6}$

7. Intra locus \& intracolor balance for all STR loci at different DNA concentrations

$\begin{array}{ll}\text { (all three injections were averaged per sample) } & \mathbf{6 7}\end{array}$

$\begin{array}{lr}\text { 8. Average sizes for FAM-labeled loci } & \mathbf{6 8}\end{array}$

9. Average sizes for JOE-labeled loci 69

10. Genotype concordance test between the LSB kit and Indentifiler kit for all

FAM-labeled loci

11. Genotype concordance test between the LSB kit and Identifiler kit for all

JOE-labeled loci

71 


\section{List of Figures}

1. Schematic of Capillary electrophoresis $\quad 23$

2. Allelic ladder used in STR typing to convert sizes into an allele call $\quad \mathbf{2 4}$

3. Internal Size Standard GS500-ROX (a) and its data points used during calibration 25

4. Artifacts commonly observed in STR profiles $\quad \mathbf{2 6}$

$\begin{array}{ll}\text { 5. Locus-Specific brackets } & \mathbf{2 7}\end{array}$

6. Illustration of D13S317 primer modifications to solve the issue of allele dropout $\quad \mathbf{2 8}$

7. Illustration of vWA primer modifications to solve the issue of allele dropout 29

8. Smaller allele at locus D13S317 recovered with the new primer 30

9. Smaller allele at locus vWA recovered with new designed primer 31

10. Optimized 5'FAM labeled multiplex 32

11. Optimized JOE labeled multiplex $\quad 33$

12. Optimized TAMRA labeled multiplex $\quad 34$

13. Optimized ROX labeled multiplex 35

14. Optimized LSB multiplex 36

15. Average heterozygote peak height ratio for the LSB multiplex 37

16. TOPO® TA Cloning® of Taq-amplified DNA

17. Illustration of primer design strategy to clone-locus specific brackets $\quad \mathbf{5 2}$

18. New FAM-labeled locus specific brackets $\quad \mathbf{5 3}$

19. New JOE-labeled locus specific brackets $\quad \mathbf{5 4}$

20. Size deviation for all FAM-labeled brackets $\quad \mathbf{5 5}$

21. Size deviation for all JOE-labeled brackets $\mathbf{5 6}$

22. LSBs allele average peak heights $\quad \mathbf{7 2}$ 
23. Heterozygous peak height ratios with various imputs of DNA concentratios

24. Size deviation for all FAM-labeled alleles based on sizes generated by ROX internal size standard

25. Size deviation for all JOE-labeled alleles based on sizes generated by ROX internal size standard 
Part One: Development of a new Multiplex for the Simultaneous Amplification of the 13 CODIS STR Loci plus Amelogenin using Locus Specific Brackets (LSBs) 


\section{Introduction}

\section{General Background on Short Tandem Repeats}

\section{$\underline{\text { Selection of the commonly used STR loci and STR Kits }}$}

More than $99.7 \%$ of the human genome does not vary between individuals; however the $0.3 \%$ that remains has been used to generate profiles that can differentiate individuals at the genetic level [1]. Certain regions of the human genome contain repeated DNA sequences, and those with core repeat units 2-7 bp in length are commonly known as microsatellites, or short tandem repeats (STRs) [2]. Thousands of polymorphic microsatellites have been found throughout the human genome but despite the high number, only a few have been chosen for the purpose of human identity testing as not all of them have proven to have the necessary characteristics that make them especially suitable for human identification [3].Such characteristics include being easily amplified by polymerase chain reaction without the problem of differential amplification; that is, given the small size of STR alleles, their PCR products are generally similar in amount, making analysis easier. STR loci have also been chosen due to their high level of polymorphism which is necessary in order to discriminate among individuals. The size of STR alleles is another advantage that makes them desirable in forensic applications. Many range from $\sim 100$ to $400 \mathrm{bp}$ in length allowing in many cases the recovery of full genetic profiles even when the DNA recovered from a crime scene is highly degraded. Their size also allows the capability of multiplexing; that is, the simultaneous PCR amplification of multiple locations of the human genome [4]

Loci with a tetranucleotide repeat are less prone to artifact alleles than shorter repeats and allow for better resolution of heterozygous alleles one repeat apart during electrophoretic analysis [5]. 
STR nomenclature is based on location. If the STR locus is within a gene, then the gene name is used for designation, e.g.TH01 within intron 01 of the human tyrosine hydroxylase gene. The name of an STR not within a gene indicates its chromosomal position, e.g. D5S818where D: DNA; 5: chromosome 5; S: single copy sequence; and 818: is the 818th locus being described on chromosome 5 [6].

STR alleles have been further categorized by repeat structure. Simple repeats contain units of identical length and sequence, compound repeats contain two or more adjacent simple repeats and complex repeats several repeat blocks of variable unit length as well as variable intervening sequences. Some tetranucleotide STR loci useful in forensics contain non-integer alleles which differ in size by only 1 or 2 nucleotides; therefore, a forensic DNA analysis instrument must permit genotyping accuracy to within 0.5 nucleotides [7].

In 1996 the Federal Bureau of Investigation solicited the advice of a wide range of forensic analysts to establish core STR loci for inclusion within the national database known as the Combined DNA Index system or CODIS (also known as the National DNA Index System, NDIS). By October of 1998 the FBI had selected 13 STR loci and it was officially launched throughout the United States. The loci chosen were D13S317, TH01, TPOX, D5S818, D7S820, D3S1358, FGA, CSF1PO, D8S1179, vWA, D16S539, D18S51, and D21S11, with amelogenin as the sex determining marker [8]. Currently NDIS contains over 6.5 million STR profiles and links all the 50 states in the United States making it possible to search criminal DNA profiles [9]. STRs are also now widely used in other areas where genotyping comparison is needed, e.g. disease research and diagnostics, parentage assessment as well as investigation of human diversity [10]. 


\section{Current approach for STR typing}

The process for STR genotyping begins by amplifying all loci through PCR. Once the DNA has been amplified the amplicon size is determined by gel or (much more commonly) capillary electrophoresis [11]. The use of fluorescent technology allows two loci with overlapping allele size ranges to be co-electrophoresed, because each can be labeled a different color. One of the PCR primers for a given locus is labeled on the $5^{\prime}$ end with a fluorescent dye. As the labeled DNA molecule passes a capillary window a laser hits the fluorophore or dye attached to the DNA fragment. [12]. The dye absorbs the energy of the laser and then emits light at a lower energy level. This energy is then converted to an electronic signal measured in relative fluorescent units (RFUs) and makes up the peaks observed on the electropherogram just like bands are observed on a gel Error! Reference source not found. [13].

The current typing method first uses an internal size standard (ISS) added to the amplified alleles prior to electrophoresis to generate a function for estimating sample allele size. The internal size standard DNA fragments span a size range larger than the sample alleles and it must be labeled with a different dye from the sample alleles, Figure 3. The sizing function relates the electrophoresis time of the known fragments, i.e. those of the internal standard and the allelic ladder.

Once the fragments have been sized each fragment gets assigned a repeat number (i.e. an allele call). For this step it is necessary to use an allelic ladder. The ladder contains most of the common alleles for each STR locus present in the population each labeled by its specific dye and it is used to calibrate the PCR product sizes to STR repeat numbers [14]. Alleles found in the ladder are PCR-amplified with the same primers provided by the STR kit to test unknown 
samples; therefore, every sample amplified by the kit will yield alleles that are identical in size as alleles present in the allelic ladder [15]. Each Allele in the ladder has been characterized by the number of repeats it contains and the range of each allele bin is $\pm 0.5 \mathrm{bp}$ around each allele; therefore, when a sample allele matches the allele from the ladder an allele call is assigned. Contrary to the internal size standard, the allelic ladder is injected separately from the samples. It is normally run within a short time period from the other sequentially processed samples alleles, whether is as part of the same batch as the samples being typed, or shortly before or after the sample set, therefore, it is assumed that it runs under the same electrophoretic conditions as all other samples being processed [16]. For analysis, the ladder is sized by the internal size standard and then the software allows the conversion from size (bp) in to a repeat number Figure 2. The most common algorithm used to determine the size of a DNA fragment is called Local Southern Method [17]. It uses two known peaks on either size of the unknown fragment being measured in order to calculate its size Figure 3. Local southern works very well for calculating sizes that range from 100 to 400 base pairs because DNA molecules in this size range have typically a linear relationship between size and time; however, because the samples and the allelic ladder have separate injections, run-to-run fluctuations in electrophoresis conditions (e.g. temperature, buffer $\mathrm{pH}$, etc.,) may cause inconsistencies in sizing reproducibility that can potentially lead to an inaccurate allele call.

STR allele calls are made in an automated fashion. However due to the presence of artifact peaks, an expert scientist is needed to double check the data. Artifacts are divided into two categories: biology related (stutter products, incomplete adenylation,) and technology related (matrix failure, dye blobs, air, and sample contaminants). 


\section{$\underline{\text { Biological related artifacts }}$}

Stutter product: is an incorrect STR allele produced by strand slippage during PCR. According to this model the newly formed strand of DNA skips one repeat unit starting the complementary base pairing with the next repeat (misalignment), pushing out a non-base pair loop from the template strand of the DNA and normally causing a deletion of one repeat unit [18].In tetranucleotides they commonly appear as one repeat shorter than the main allele (n-4) but they can also be observed as one repeat larger (non-base pair loop inserted) than the main allele $(n+4)$

Figure 4a. The acceptable upper limit for stutter is 3SD above the highest stutter percentage normally observed at that locus or a general value of $15 \%$ [19]

Incomplete adenylation: incomplete adenylation is the result of PCR amplification with too much DNA template. During the PCR reaction the Taq polymerase catalyzes the addition of an extra nucleotide (adenine) at the 3' end of the PCR product. However when this fails to happen split peaks are produced known as $-\mathrm{A}$ and $+\mathrm{A}$. Because one of the alleles will be off ladder, manual edition by the expert is needed Figure $\mathbf{4 b}$ [20].

\section{$\underline{\text { Technology related artifacts }}$}

Matrix failure: known as pull up, matrix failure results when the instrument fails to resolve the dye colors. The spectral overlaps occur for examples when a sample is overloaded and it exceeds the linear range of detection. The result is peaks of $>1$ color for a single fragment, or a highly elevated baseline for any given color [1].

Dye blobs: dye blobs occur when the fluorescent molecule detaches itself from the primer and migrates through the capillary independently. Dye blob peaks are typically broader than an allele [21]. 


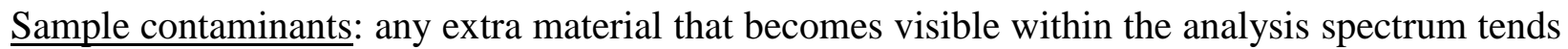
to interfere with the results as they appear as peaks. Many contaminants appear as broad peaks that can be distinguished from the true alleles [22].

\section{Additional peaks present in STR Profiles}

Triallelic pattern: rare individuals show three alleles at a single locus instead of two. One explanation is a somatic mutation of an allele, resulting in a chimera with some cells containing the original alleles and some cells containing the mutant allele. This produces uneven peak heights for two of the affected alleles that when added together are equal in height as the normal allele. The second type occurs as a localized duplication or chromosomal aneuploidy producing peaks that are all similar in heights Figure 4c [23].

Mixed sample results: a mixture of $>1$ individual is apparent when multiple loci have more than two alleles of equal intensities at a particular locus or severe heterozygous imbalance. Determining the individual genotypes in a mixture is particularly difficult if one of the contributors is, $1 / 10^{\text {th }}$ of the major contributor, or if there are multiple individuals [24].

\section{Advantages of Multiplex PCR}

Multiplex PCR, the amplification of several loci within the same PCR reaction allows for the efficient use of sample DNA and reduces the possibility of contamination or data mix-up compared to many separate reactions [25]. After the 13 core loci for CODIS were adopted in the United States, Life technologies (Applied Biosystems) and Promega Corporation marketed multiplex STR kits for typing some or all CODIS loci plus amelogenin [26]. Common problems 
in PCR-based STR analyses are the production of false negatives due to reaction failure or false positives due to contamination of the PCR reaction. In multiplex assays each amplicon provides an internal positive control for the other loci. A failure to amplify one but not all loci, points to incomplete template degradation rather than complete degradation or a problem with the reaction mix. An external (separate tube) positive control is still needed to monitor conditions such as thermal cycler function [27].

\section{Factors affecting DNA mobility during electrophoresis}

Whether the separation occurs on gel or capillary the basic principle is the same. Molecules are separated on the basis of their rate of movement through a gel/capillary under the influence of an electrical field. Electrophoretic mobility is a function of DNA fragment size and shape, the density of the gel/polymer, temperature, buffer, and the strength of the electrical field [13]. During electrophoresis DNA interacts efficiently with the sieving polymer, and its size is proportional to its length, a relationship that is the key to accurate genotyping by CE. [28]. Denaturing conditions are maintained by chemicals such as urea, formamide, and 2pyrrolidinone, but even under strong denaturing conditions DNA can assume its secondary structure, e.g. a loop/hairpin. For this reason high temperature is also used to promote denaturation [29].

\section{$\underline{\text { Commercial Control of Life Science Products and Automated Platforms }}$}

The current technology surrounded STR technology has been for decades dominated by two major companies in the United States, Applied Biosystems (AB) and Promega Corporation. These two companies hold the vast majority of the market from kits, reagents and software to the 
integrated platforms that are currently in almost every forensic lab in the United States [30]. As the demands of generating, entering and maintaining the DNA profiles in the national database increases, so does the need for new automation that can increase sample throughput, reduce cost and allow for full resolution of STR base composition. Anew approach to allow this type of resolution has been developed by Roche laboratories. The Roche genome sequencer FLX platform has been used to simultaneously sequence multiple core STR loci and directly compare these sequences to conventional CE-based STR technology. This new method is advantageous as it reveals DNA base substitutions and repeat sequence variations that would not have been identified with the current STR typing method [31]. Another new significant development has been the combination of PCR with $\mathrm{CE}$ on a microchip to produce a fully integrated and automated STR analysis system. The advantage of such a system would increase throughput separation of forensic samples increase workflow and lower the costs by reducing the electrophoretic analysis from submicroliter to nanoliter sample volumes [32].

\section{$\underline{\text { STR Typing with Locus-Specific Brackets (LSBs) }}$}

Locus Specific Brackets were developed as an alternative sizing method for detecting STR fragment length. LSBs are internal size standards that flank each targeted locus. They are artificial alleles created to be 1 or more repeat units shorter or longer than the common alleles of an STR locus but having identical (or very similar) flanking regions Figure 5 [33]. As the sample allele and LSB sequences are nearly identical, the electrophoretic behavior is expected to be nearly identical. Therefore the sizing function should be much more robust between-injection variation in eletrophoretic conditions compared to sizing using an ISS. [34]. Just as in any other STR kit, alleles can fall outside the desired range. If shorter or longer than its respective brackets 
it will migrate between sets of LSBs from adjacent loci (equivalent to an off-ladder allele in the conventional method); however, if an allele were to be exactly the same size and sequence as the bracket, the true allele would be masked by the LSB and the genotype would be incorrect e.g. a heterozygous locus would appear as homozygous. Just like the conventional approach Locus Specific Brackets utilizes the local southern method to determine the size of the unknown DNA fragments by using the reciprocal relationship between fragment length and mobility.

Other potential advantages of Locus Specific Brackets over conventional human STR kits are that the same number of loci requires one fewer dye per kit; that used for the ISS, and the lack of an allelic ladder means every injection can yield a sample profile. Therefore an LSB kit could reduce costs and analysis time compared to the kits currently in use.

\section{Y-STR Typing with Locus Specific Brackets}

Locus-Specific Brackets were found to be reliable for Y-chromosome STR typing. Potential problems compared to a conventional kit discussed by the authors include masking of a sample allele by an LSB and the fact that new sample fragment sizes cannot be developed for the LSB size ranges [35].

\section{Preliminary Validation of the LSB Multiplex}

The now defunct company known as Oligotrail, LCC, developed an LSB multiplex for the 13 CODIS STR loci plus amelogenin [36]. Prior to the current study, members of our research lab attempted to validate this kit by measuring concordance with profiles from the same human 
subjects produced by the commercial kit identifiler $(\mathrm{AB})$. The reagents, manual and genotyping software were supplied by a former Oligotrail research scientist.

99 human subjects were profiled using both kits for a total of 1330 allele comparisons (unpublished). 1315 alleles were concordant 14 of the samples amplified with the LSB kit had allele dropout at loci D13S317 and vWA. DNA sequencing of these individuals revealed a primer annealing site polymorphism for D13S317, a likely explanation for the allelic dropout. One LSB vWA primer site overlapped the repeat region, also a likely explanation for the dropout. Oligotrail had not encountered these problems, perhaps because they relied on a geographically limited selection of research subjects (East Asian), while our study was a mixture of ethnicities.

I took over the project to make a new reaction (LSB mixes reagents were getting scarce), and to design new primers to solve the dropout problem and include them in the new mix. During the course of my preliminary work I encountered unexpected challenges that changed the original course of the project. Almost all fragment sizes generated by the a reaction mix I made with primer sequences from the Oligotrail genotyping software (called Oligocode) manual were in disagreement with fragment sizes from the previous validation study (same sample). Further investigation revealed that primer sequences given in the LSB manual were not the same as the primers present in the LSB multiplex used during the previous validation study (current and original LSB sequences are given in appendix Table 1). Due to the observed differences in primer annealing sites, the new mix faced a potential overlap between adjacent loci of the same color, and a [new] reverse primer site included a polymorphism that could potentially create locus imbalance or allele dropout. Shifts in fragment size also meant that profiles would not be correctly genotyped with Oligocode. 
Because at this point the optimization of the new LSB mix was very advanced, and primers designed to solve dropout issues had already been included in the new mix, I opted to keep the current LSB multiplex, design new primers for affected loci and synthesize new brackets.

\section{$\underline{\text { Goal }}$}

The goal of this project was to produce an LSB-based human STR multiplex reaction kit, free of allelic dropout and with appropriate LSB size standards for analysis by the Oligotrail genotyping software, and to do a simple performance evaluation of the kit by testing the reproducibility of profiles and the sensitivity of the reaction. 


\section{Materials \& Methods}

\section{DNA Sample preparation}

DNA samples were already extracted and quantified as recommended by SWGDAM revised validation guidelines during the previous validation of the LSB kit [37]. Liquid blood samples (100ul) and buccal swabs were collected from 100 anonymous (WVU IRB protocol 16279). DNA extraction from the liquid blood samples was done using Qiagen (Valencia, CA) DNeasy kit according to protocol for whole nucleated blood, with a final elution of 100uL. Buccal swab samples were extracted using the Qiagen DNeasy kit according to manufacturers' directions for DNA extraction from tissues, with a final elution of $100 \mathrm{uL}$. All samples were quantified for nucleic acid content using the nanodrop spectrophotometer and further diluted to a working concentration of $1 \mathrm{ng} / \mu \mathrm{l}$.

\section{$\underline{\text { LSB-Multiplex Primer design \& synthesis }}$}

All primes were designed using the Oligoanalyzer tool from Integrated DNA Technology, and only primers that fit the best criteria (e.g. specificity, melting temperature, GC content, hairpin structure, self-dimer-structures etc.) for ultimate performance were chosen [38]. All primer lengths were between $18-30$ bases to maintain high sequence specificity provided that the annealing temperature was optimal. The GC content was kept between 40-60 percent, allowing for a melting temperature between $54^{\circ} \mathrm{C}-58^{\circ} \mathrm{C}$. HPLC purified 5 ' fluorescently labeled primers were purchased from Operon Biotechnologies, Inc (Huntsville, AL) containing either FAM (carboxyfluorescein), JOE ( $\beta$-carboxy-4',5'-dichloro-2',7'-dimethoxyfluorescein) TAMRA (N,N,N',N'-tetramethyl- $\beta$-carboxyrhodamine), or $\operatorname{ROX} \quad(\beta$-carboxy-X-rhodamine). Nonfluorescently labeled primers were purchased from Integrated DNA Technologies (Coralville, 
IA) and the method of purification was standard desalted (primer sequences are given in table

\section{1 of appendix).}

D13S317: A new unlabeled forward primer was designed containing the nucleotide present in the sample with the mutation (Table1). The original and new primers were combined at equal ratios to act as a degenerate primer that will bind whether a $\mathrm{T}$ or a $\mathrm{C}$ is present and were tested on a sample for which dropout was previously observed and the 5'label was transferred from the forward to the reverse primer (Figure 6).

vWA: A new unlabeled reverse primer (vWA-RLS) was designed to avoid the overlap between the primer and the repeat region by shifting the primer binding site 9 base pairs away from the repeat region (Figure 7). Again, the primer was tested on a sample for which dropout was observed (Table 1)

D18S51: A new unlabeled reverse primer was designed for this locus because the smallest allele generated by the Oligotrail primer falls on the wrong side the long Bracket of D21S11. The primer was shifted 46 bp away from the repeat region. The primer sequence is given in Table 1.

CSF1PO: A new unlabeled reverse primer was designed for this locus because the Oligotrail primers yield an allele size range overlapping the long bracket of D16S539. The primer was shifted 29 bp away from the repeat region. The primer sequence is given in Table 1.

\section{$\underline{\text { Test for Signal Strength in Monoplex PCR reactions }}$}

For initial testing purposes all primers were diluted to a final concentration of 5pmol/uL. Each locus was amplified individually under the PCR reaction conditions and Thermal Cycler parameters described below (see section on PCR amplification), to ensure that the correct size product was generated. 


\section{Optimization of each single dye multiplex}

After all primer pairs where individually tested, all loci labeled with the same dye were combined. At this point the entire multiplex was still divided into four reactions. These were the FAM-labeled triplex of D13S317, TH01, and TPOX; JOE-labeled tetraplex of Amelogenin, D5S818, D7S820 and FGA; TAMRA-labeled triplex of vWA, D21S11 and D18S51, and ROXlabeled tetraplex of D3S1358, D8S1179, D16S539, and CSF1PO.

Peak height was balanced by increasing or decreasing primer concentration for an individual locus depending on whether the peaks were too low or too high (Primer concentrations are given in Table 2 of appendix).

\section{$\underline{\text { Multiplex optimization (all colors) }}$}

Once optimization was done by color, the process was repeated with all colors combined. This involved extensive trial and error, because the more primers combined, the less predictable the optimization process becomes as increased competition between the primers produces nonlinear relationships between primer concentration and peak height (Primer concentrations are given in Table 3 of appendix).

\section{$\underline{\text { PCR amplification }}$}

For all single locus amplifications each PCR reaction included $3 \mu \mathrm{L}$ of $5 \mathrm{X}$ LSB buffer (Tris $\mathrm{HCl}$ 67Mm, $\mathrm{Ph} 8.3, \mathrm{KCl} 333 \mathrm{Mm}, \mathrm{MgCl}_{2} 10 \mathrm{Mm}$, gelatin $\left..007 \%\right)$ and $3 \mu \mathrm{L}$ of $5 \mathrm{X}$ dNTPs mix $(25 \mathrm{mM}$ each from Promega Corporation, Madison, WI). The 5U Immolase Taq polymerase from Bioline USA Inc, was diluted to $1 \mathrm{U}$ and one microliter was used per reaction. $2 \mathrm{uL}$ of each 5 pmol primer was used together with $3 \mathrm{uL}$ of water and 1ng DNA template for a total of $15 \mathrm{uL}$. For single dye 
and full multiplex reactions $1.5 \mathrm{uL}$ of primer mix were used, $1.5 \mathrm{ul}$ of $10 \mathrm{X}$ dNTPs and $1 \mathrm{uL}$ of $5 \mathrm{U}$ of Taq polymerase were used instead. All samples were amplified on a Perkin-Elmer Thermal Cycler Model 9700 with the following cycling parameters $95^{\circ} \mathrm{C}$ for 10 minutes, then 10 cycles of $94^{\circ} \mathrm{C}$ for 30 seconds, (touchdown) $71-60^{\circ} \mathrm{C}$ for 30 seconds $(30 \% \mathrm{rmp}), 72^{\circ} \mathrm{C}$ for 60 seconds (20\% rmp), then 26 cycles of $94^{\circ} \mathrm{C}$ for 30 seconds, $59^{\circ} \mathrm{C}$ for 30 seconds ( $30 \% \mathrm{rmp}$ ), $72^{\circ} \mathrm{C}$ for 60 seconds (20\%rmp), and a final extension of 60 minutes at $70^{\circ} \mathrm{C}$.

\section{$\underline{\text { Capillary Electrophoresis }}$}

1uL of amplified PCR product and 0.5uL of ROX-500 [39] size standard were combined with 15uL of HiDi foramide (Applied Biosystems). This mixture was denatured at $95^{\circ} \mathrm{C}$ for 3 minutes followed by snap cooling on ice for 3 minutes. Samples were then loaded onto the ABI Prism 310 Genetic analyzer (AB) using a 60cm capillary and POP-4 polymer (Applied Biosystems). Injection was done at $15.0 \mathrm{kV}$ for $5 \mathrm{sec}$, with a run temperature of $60^{\circ} \mathrm{C}$ and a run time of 30 minutes per sample.

\section{$\underline{\text { Peak Height Ratio (PHR) }}$}

Intra-locus peak height ratios (PHR) were calculated for all 13 loci by dividing the peak height of an allele with a lower RFU value by the peak height of an allele with a higher RFU value, and then multiplying this value by 100 to express the value as percentage. The ratio was calculated using 3 samples, at DNA concentrations of 1, 0.5, 0.1, and 0.01 nanograms [40]. 


\section{Stutter}

Stutter percentages were calculated by dividing the height of the stutter peak by the height of the allele [19]. Stutter was not calculated in some loci as the level of noise in the electropherorograms obscured any stutter, and in others stutter was simply not observed. 


\section{$\underline{\text { Results }}$}

Allele dropout from previous LSB work

The new primers successfully amplified alleles at locus D13S317 (genotype 9, 11) and vWA (14, 18) that displayed dropout in the earlier multiplex (Figure 8 \& 9 respectively).

Size overlap

Primers designed to shift the size ranges of D18S51 and CSF1PO away from the LSB of an adjacent locus failed to amplify. Therefore the multiplex was prepared with the original primers for these loci.

\section{Test for Signal Strength in Single Locus PCR reactions}

All 13 STR loci individually amplified were detected and separated through the ABIPrism -310 Genetic analyzer, and sized with ROX-500. Every peak was above 1000 RFUs, and differences among heterozygous allele sizes were concordant with identifiler profile from the same sample confirming the specificity of the LSB primers.

Multiplex optimization (single dye only)

After a series of PCR reactions where primer titrations were constantly being adjusted a relatively balanced multiplex was produced for each color Figure 10-13. In general the increase/decrease of peak height was unpredictable. However, in many cases the balance was improved by simply increasing the primer concentration of the larger fragments while keeping the concentration of smaller fragment the same or by reducing the primer concentrations of the smaller fragments and increasing the primer concentrations of the larger ones. 


\section{Multiplex optimization}

At this step titration of 29 primers became a lot more complicated as more primers competed for reagents. The titration process was the same as the previous step and after a thorough optimization a relatively balanced multiplex was obtained Figure14.

\section{LSB Multiplex PHR}

The peak height ratio for the multiplex was calculated based on the data generated by GeneScan software (AB), for all heterozygous loci present in all three samples. At 1ng all 12 heterozygous loci present in all three samples presented peak height ratios above 50\% except locus TPOX. With a lower concentration of $0.5 n g$ Locus D5 showed a PHR of $45 \%$ in two samples for all injections and TPOX imbalanced increased with a PHR of $17 \%$ in one sample and $8 \%$ in another. With 0.1 ng of DNA template more loci presented a PHR of less than $50 \%$ such as amelogenin, D7S820, D8S1179 D3S1358, and D5S818 with ratios between 40 and 50 percent, and D16S539 and D21S11 with ratios between 35 and 40 percent. At this concentration TPOX experienced allele dropout. At $0.001 \mathrm{ng}$, the entire multiplex experienced severe allele imbalanced in all loci with the majority of loci with ratios below 50\%. Many loci also experienced allele dropout Figure 15. Although the allele imbalance at both 0.5 and 0.1 nanograms of template is less than $50 \%$, all allele heights were above 400 relative fluorescent units (RFU).

\section{Stutter percentage}

Stutter peaks were not observed in D13S317, TH01, TPOX, amelogenin, CSF1PO and for D5S818, D8S1179 and D16S539 the high background noised made it impossible to accurate 
measure stutter peaks. Stutter percentage was calculated for loci D7S820, FGA, vWA, D21S11, D18S51 and D3S1358. High percent stutter was found for allele 10 in locus D7S820 (22\%), allele 17 in locus D3S1358 (32\%) and alleles 33.2 and 29in locus D21S11 (26\% \%21\%) respectively. All other alleles were below the $20 \%$ range (Table 2). 
Table 1. New primers for D13S317, vWA, D18S51 \& CSFIPO STR loci. These primers were designed to resolve the allelic dropout, and fragment overlap issues

\begin{tabular}{lll}
\hline Locus & \multicolumn{1}{c}{ Sequence $\left(\mathbf{5}^{\prime} \longrightarrow \mathbf{3}^{\prime}\right)$} & Length $(\mathbf{b p})$ \\
\hline $\begin{array}{l}\text { D13S317 } \\
\text { Forward } \\
\text { vWA-RLS } \\
\text { Forward }\end{array}$ & ACT CTG ACC CAT CTA ATG CCT A & 22 \\
$\begin{array}{l}\text { D18S51.R } \\
\begin{array}{l}\text { Forward } \\
\text { CSF1PO.R } \\
\text { Forward }\end{array}\end{array}$ & ACAGATGATAAATACATAGGATGGATGG & 28 \\
\hline
\end{tabular}


Table 2. Stutter percentage on various STR loci with LSB multiplex

\begin{tabular}{|c|c|c|c|c|}
\hline Loci & Allele & Sample & Average & SD \\
\hline D7S820 & 10 & B483 & $22 \%$ & $3.16 \%$ \\
\hline FGA & 23 & B483 & $13 \%$ & $2.43 \%$ \\
\hline FGA & 18 & B910 & $11 \%$ & $0.66 \%$ \\
\hline FGA & 21 & B910 & $12 \%$ & $2.35 \%$ \\
\hline FGA & 19 & BKA & $7 \%$ & $2.33 \%$ \\
\hline VWA & 17 & B483 & $11 \%$ & $1.17 \%$ \\
\hline VWA & 18 & B910 & $12 \%$ & $0.04 \%$ \\
\hline D21S11 & 29 & B483 & $21 \%$ & $11.05 \%$ \\
\hline D21S11 & 33.2 & B910 & $26 \%$ & $0.32 \%$ \\
\hline D21S11 & 30 & BKA & $14 \%$ & $2.39 \%$ \\
\hline D18S51 & 16 & B483 & $16 \%$ & $1.00 \%$ \\
\hline D18S51 & 14 & B910 & $19 \%$ & $5.63 \%$ \\
\hline D3S1358 & 17 & B483 & $32 \%$ & $7.67 \%$ \\
\hline D3S1358 & 15 & B910 & $11 \%$ & $1.88 \%$ \\
\hline
\end{tabular}




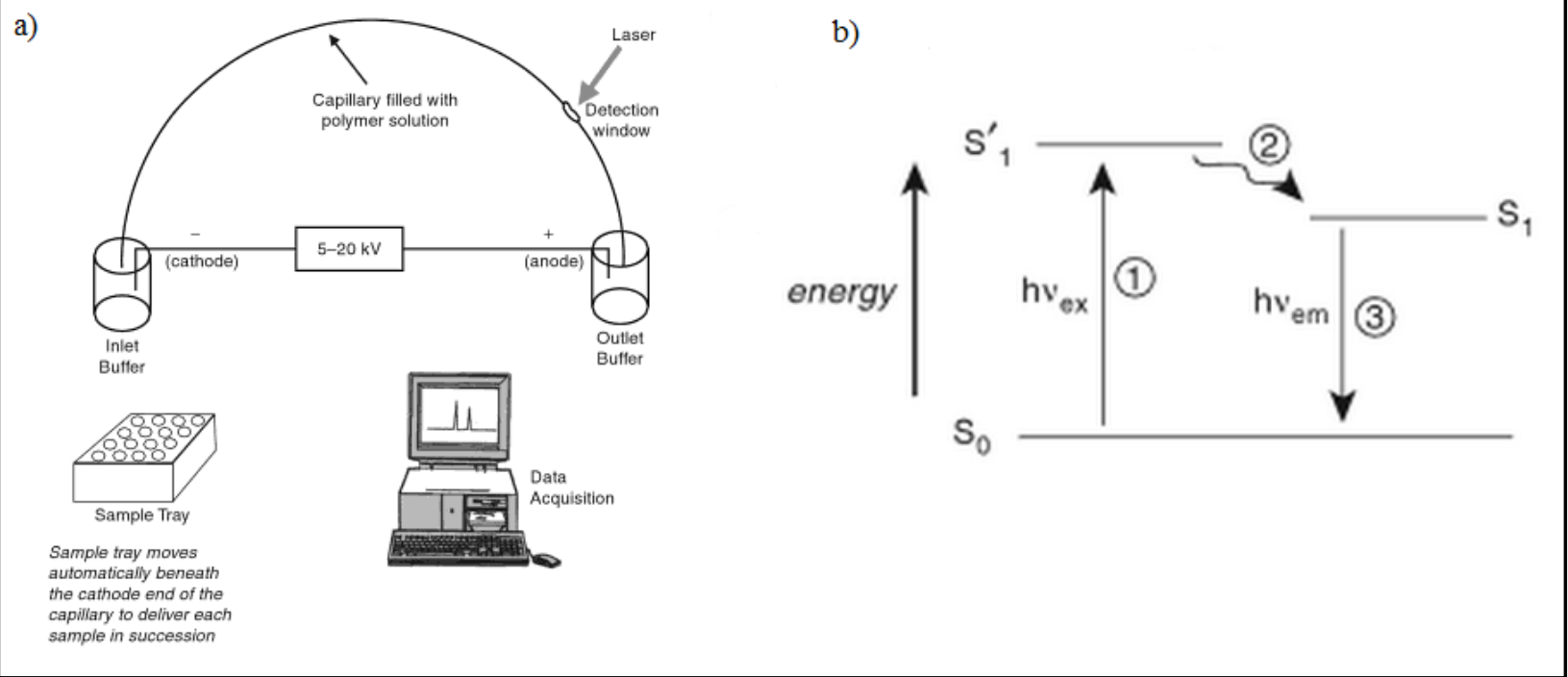

Figure 1: Schematic of Capillary electrophoresis (a) and fluorescence process (b). Samples are loaded into a tray and injected into polymer-filled capillary by applying high voltage. As the fluorescently labeled samples pass through the detection a laser beam hits the dye labeled molecule (a). The laser beam excites the flurophore from its ground state to an excited transitions state to eventualyl release a photon at a lower energy level hence (b) [1]. 


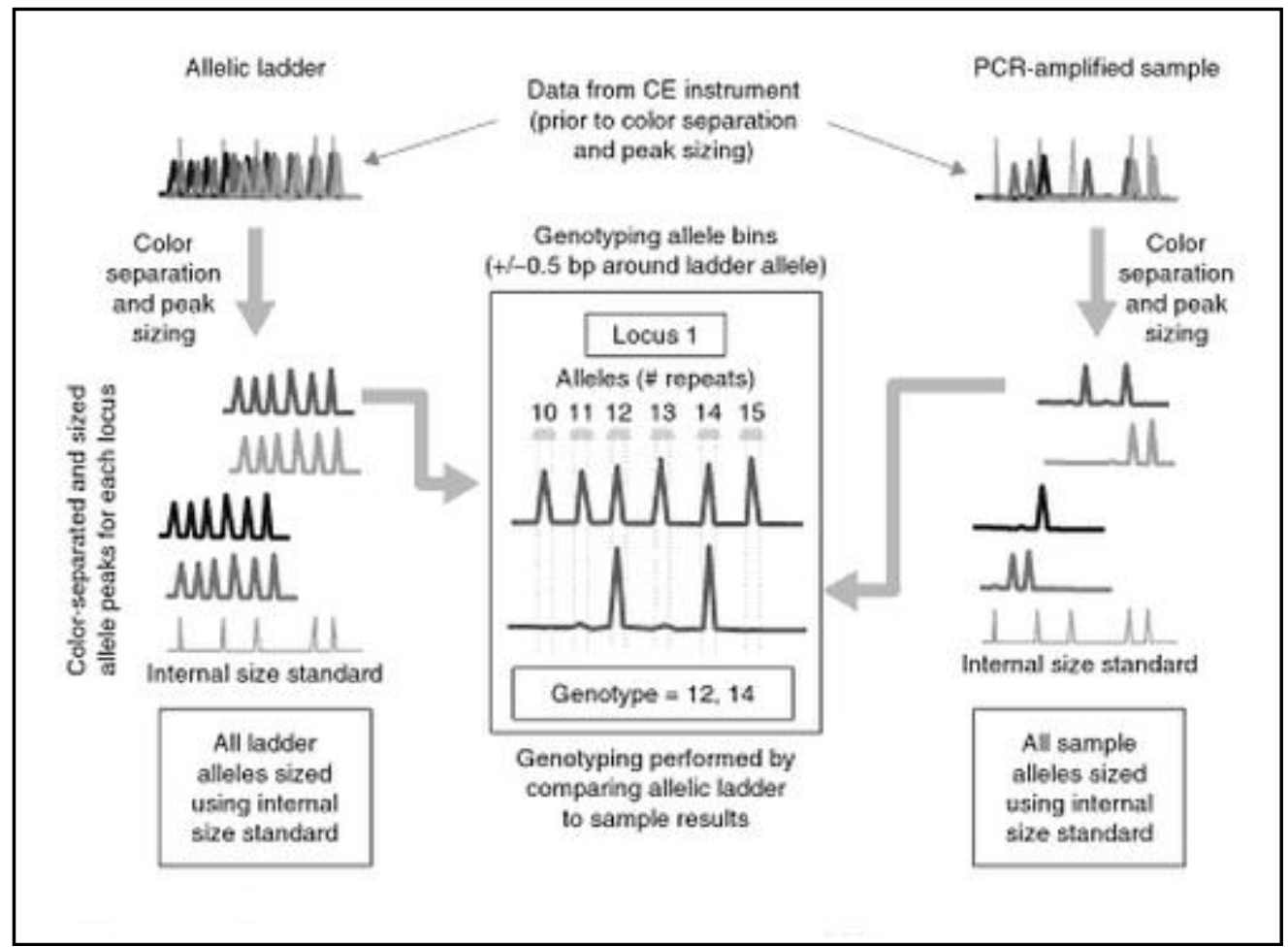

Figure 2: Allelic ladder used in STR typing to convert sizes into an allele call. Just like the alleles of unknown samples, all alleles present in the allelic ladder for each locus are sized by the internal size standard for comparison.) [1]. 


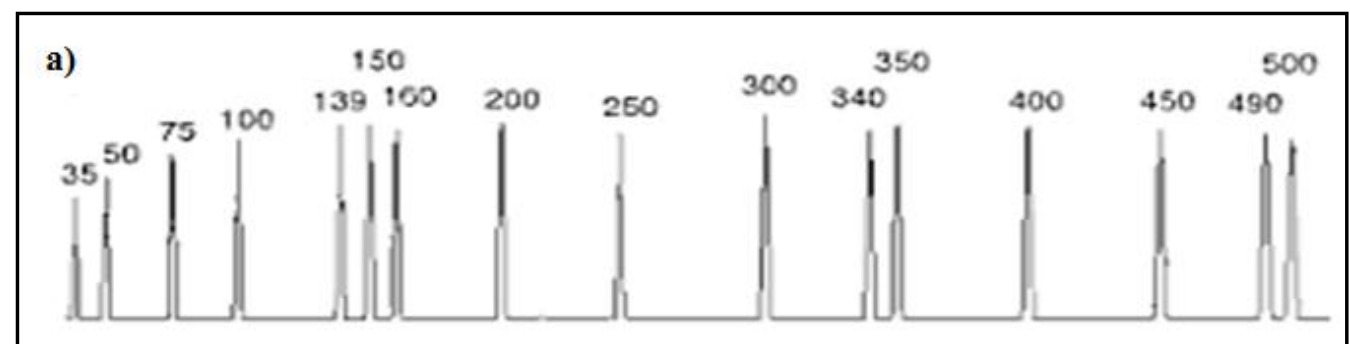

b)

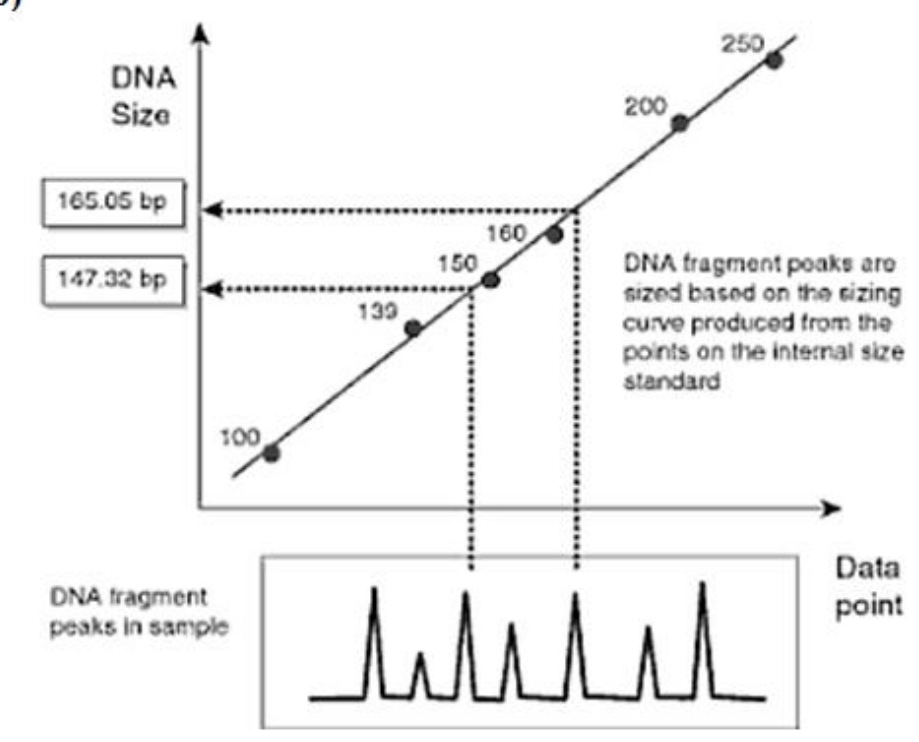

Figure 3: Internal Size Standard GS500-ROX (a) and its data points used during calibration. The internal size standard contains DNA fragments of various lengths (a) and is used to size both the allelic ladder and unknown samples (b) [1] 


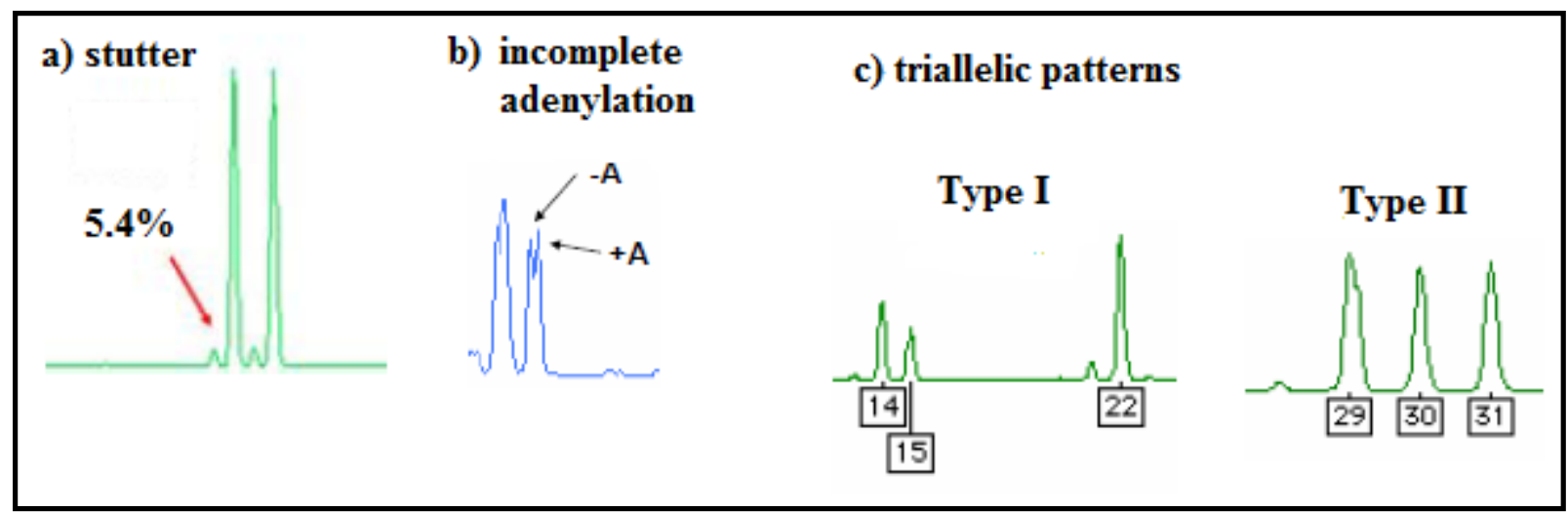

Figure 4: Artifacts commonly observed in STR profiles. Their identification is necessary as they can interfere with the analysis, especially mixture [41]. 


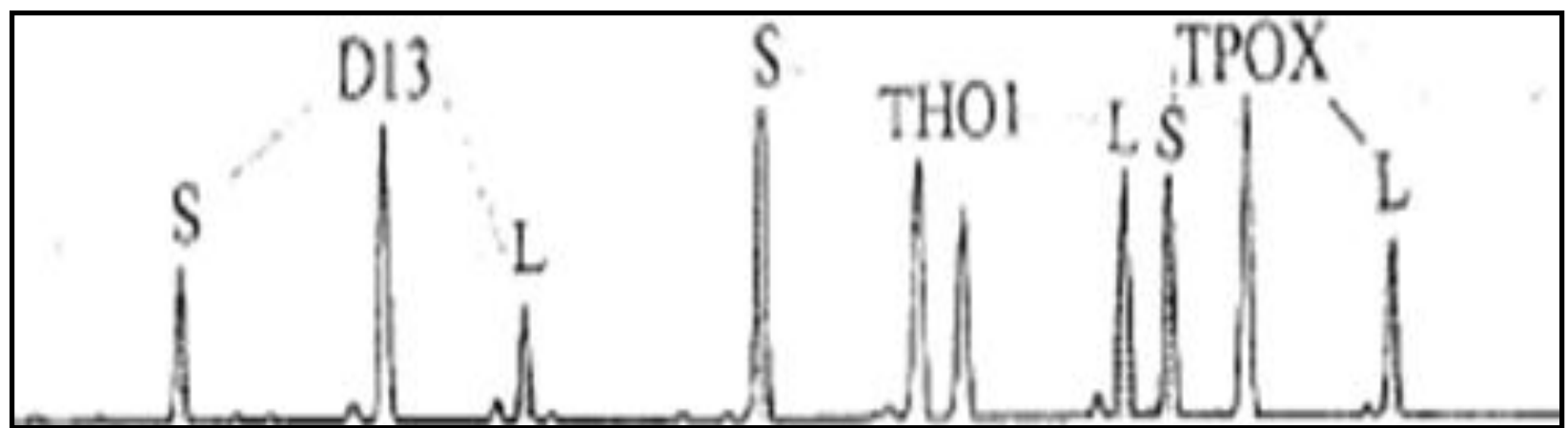

Figure 5: Locus-Specific brackets. A short (S) and a long (L) bracket is used to size and genotype each locus by making the brackets one or more repeats shorter/longer than the smallest/larger common allele for each locus [33]. 
Old

D13S317 Primer Modifications

F. Primer

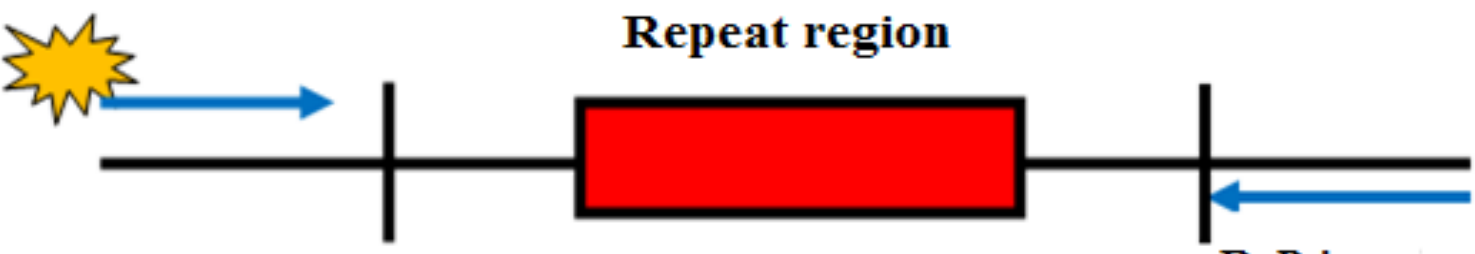

R. Primer

New

F. Primer

Repeat region

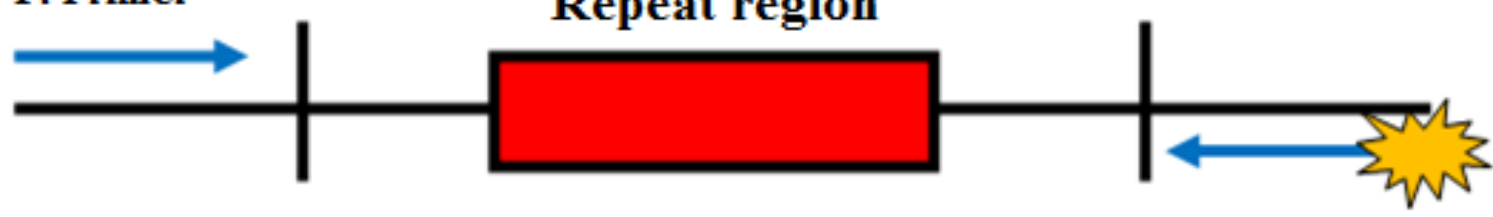

R. Primer

Figure 6: Illustration of D13S317 primer modifications to solve the issue of allele dropout. Arrows indicate the directions of the primer. Labeled has been switched from the forward primer to the reverse primer. 


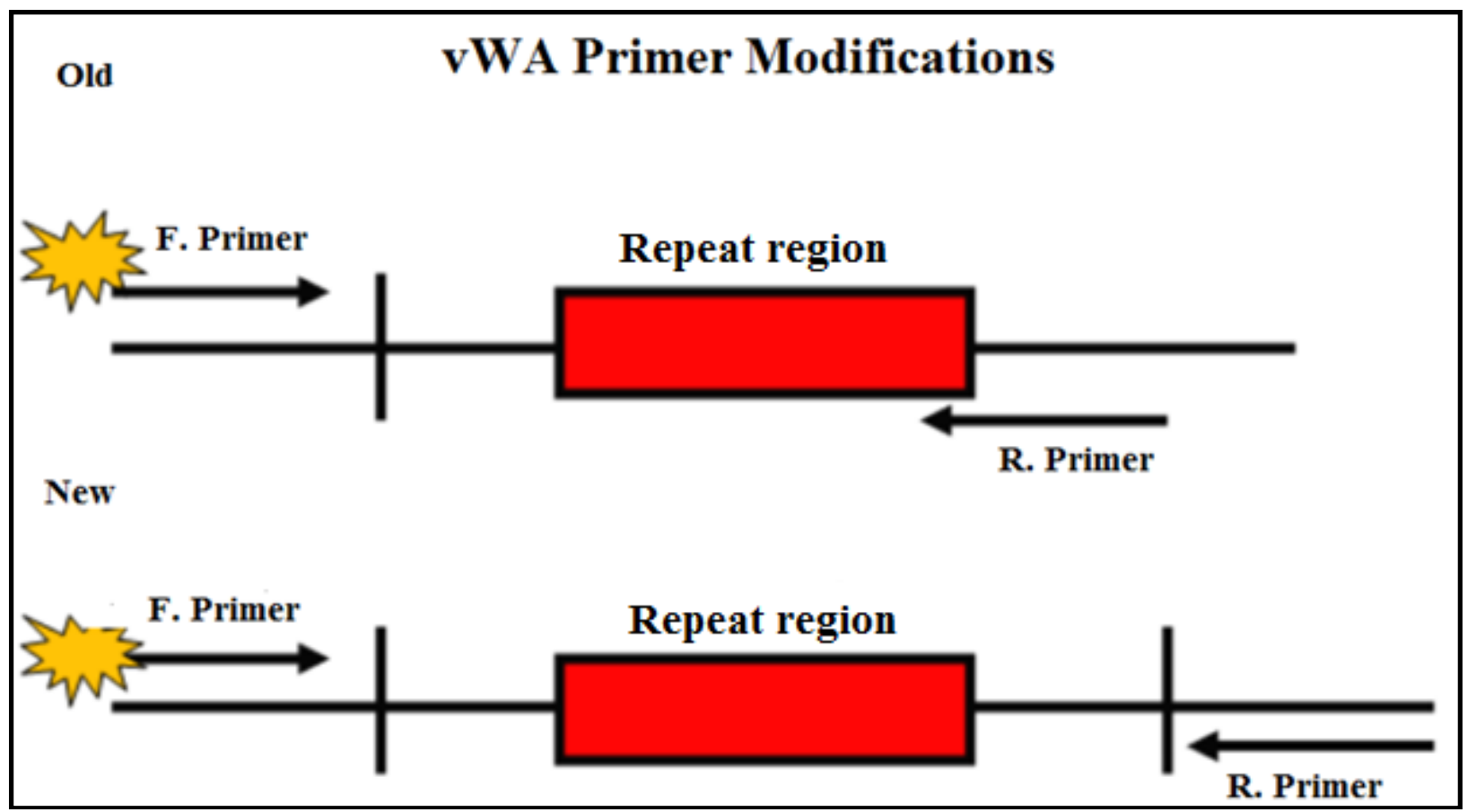

Figure 7: Illustration of vWA primer modifications to solve the issue of allele dropout. The arrows indicate the position of the primer. The new reverse primer is shown shifted away from the repeat region in order avoid overlap. 


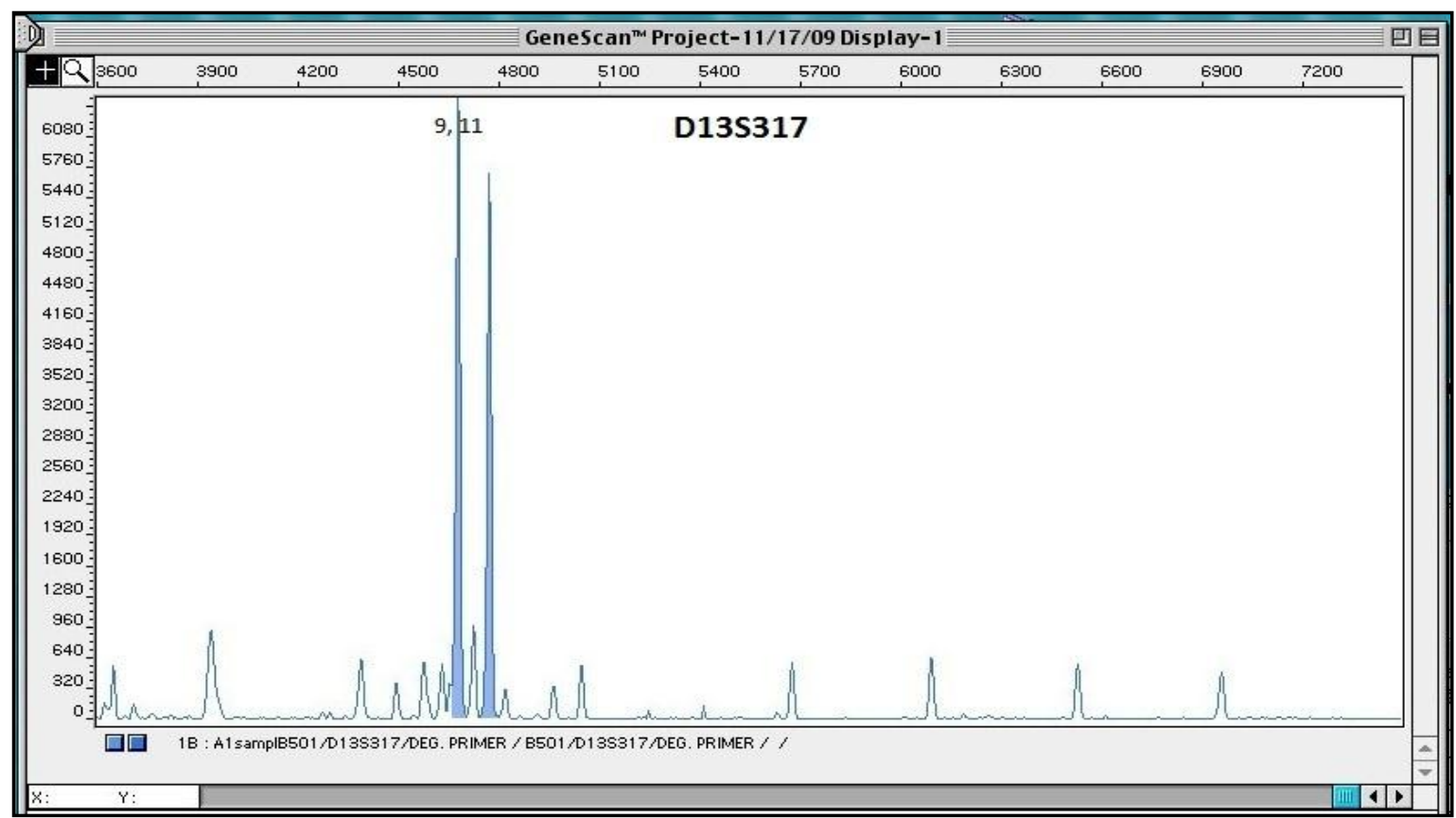

Figure 8. Smaller allele at locus D13S317 recovered with the new primer 


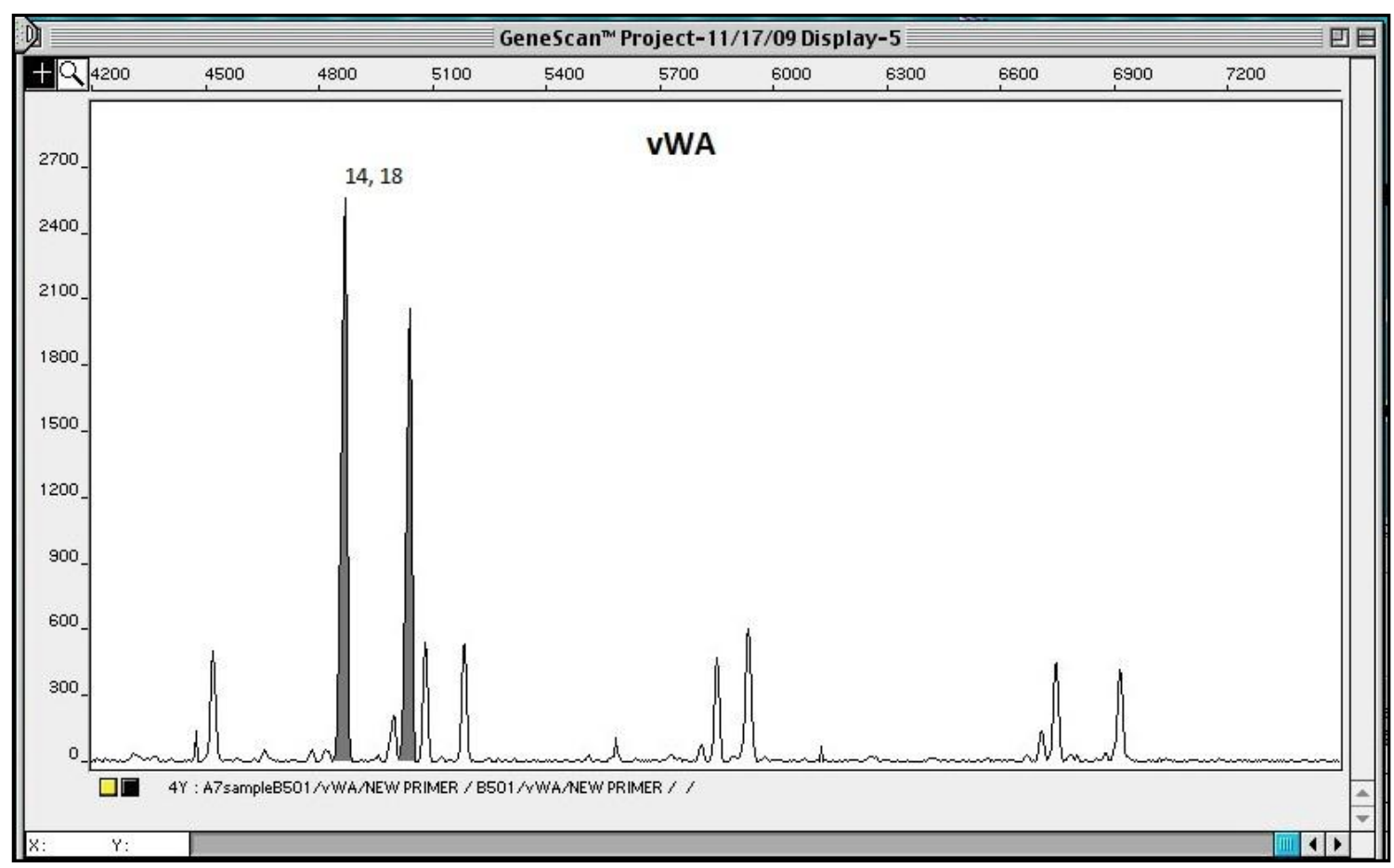

Figure 9. Smaller allele at locus vWA recovered with new designed primer. 


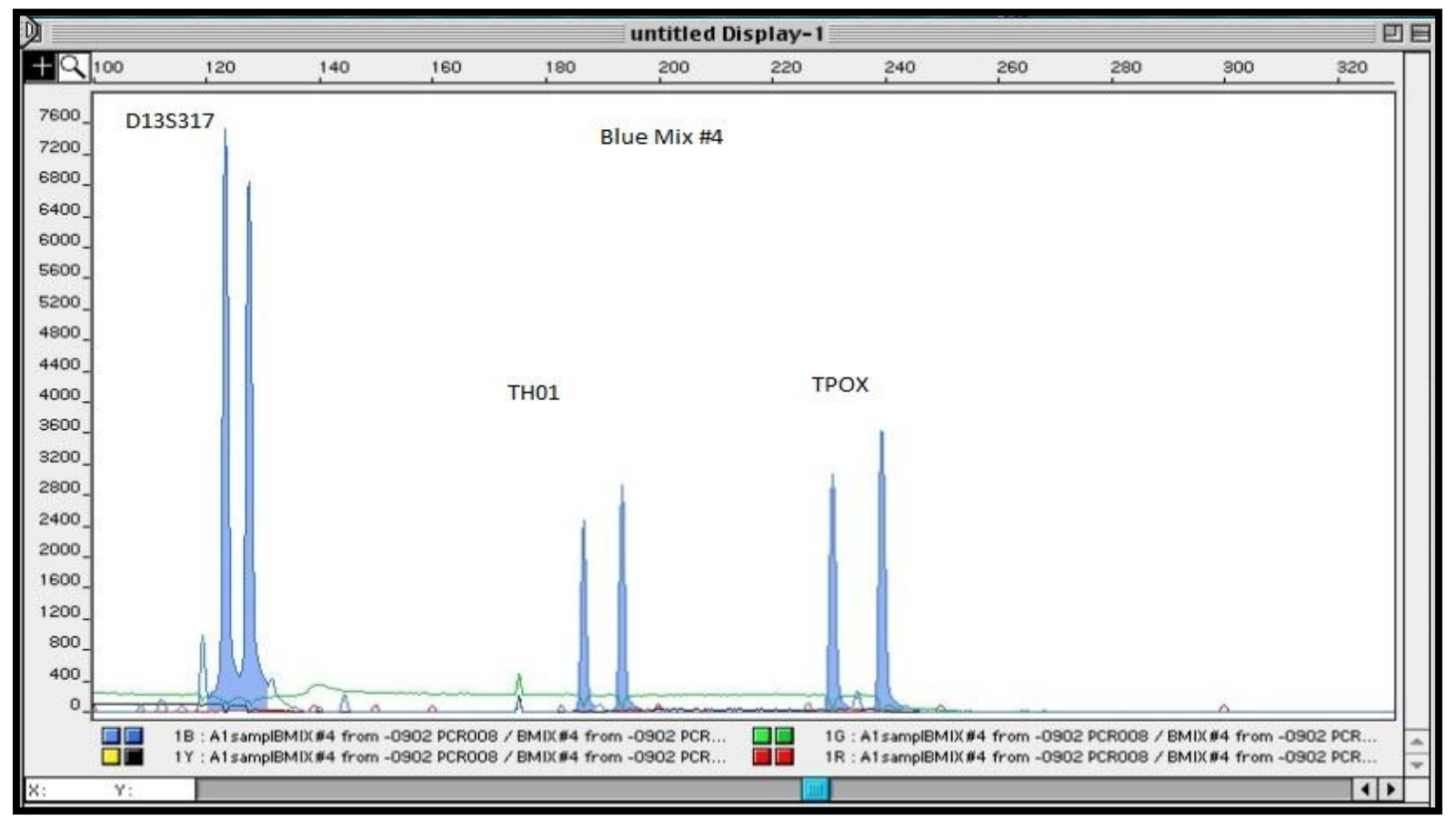

Figure 10. Optimized 5'-FAM labeled multiplex. 


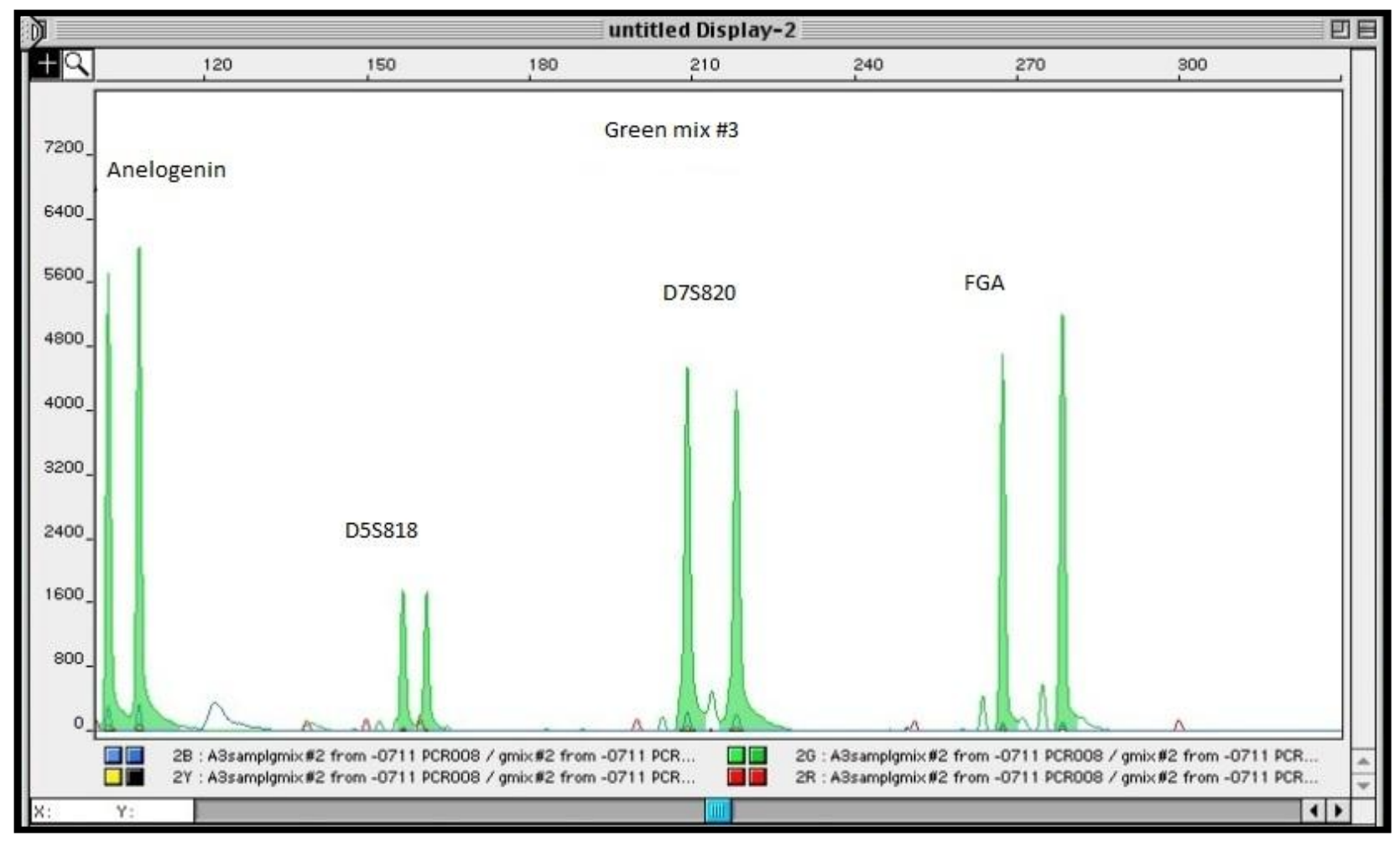

Figure 11. Optimized JOE labeled multiplex. 


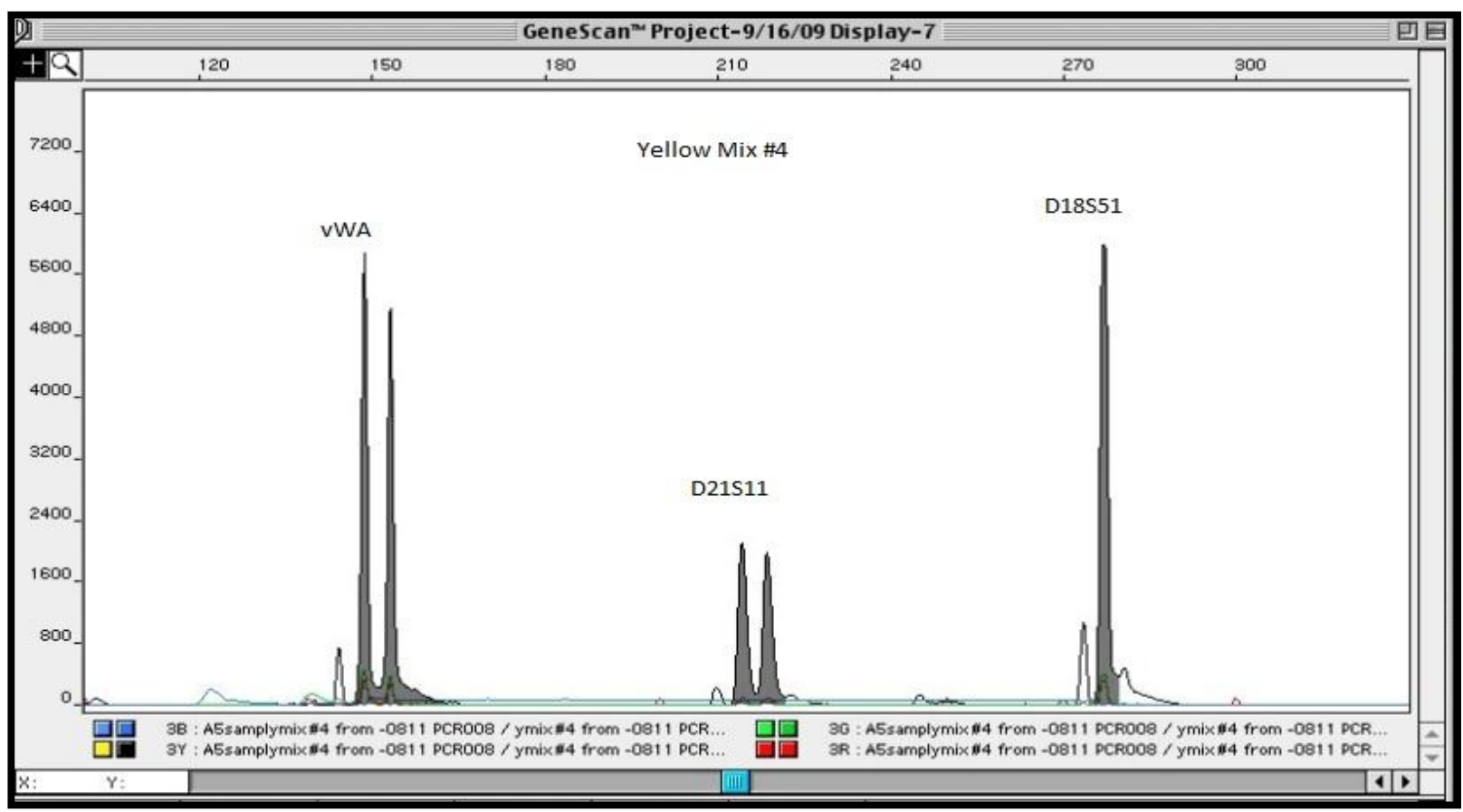

Figure 12. Optimized TAMRA labeled multiplex. 


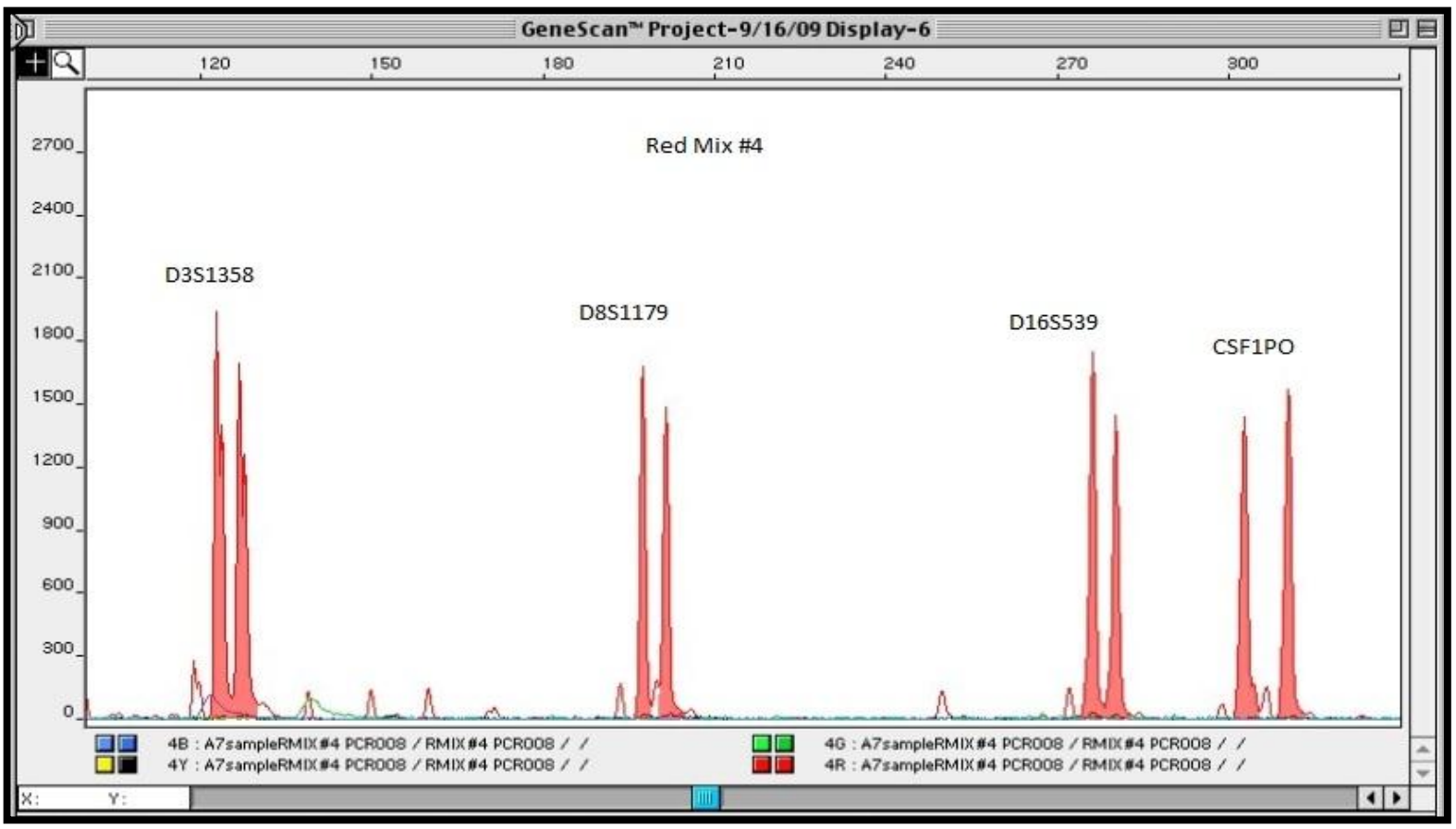

Figure 13. Optimized ROX labeled multiplex. 


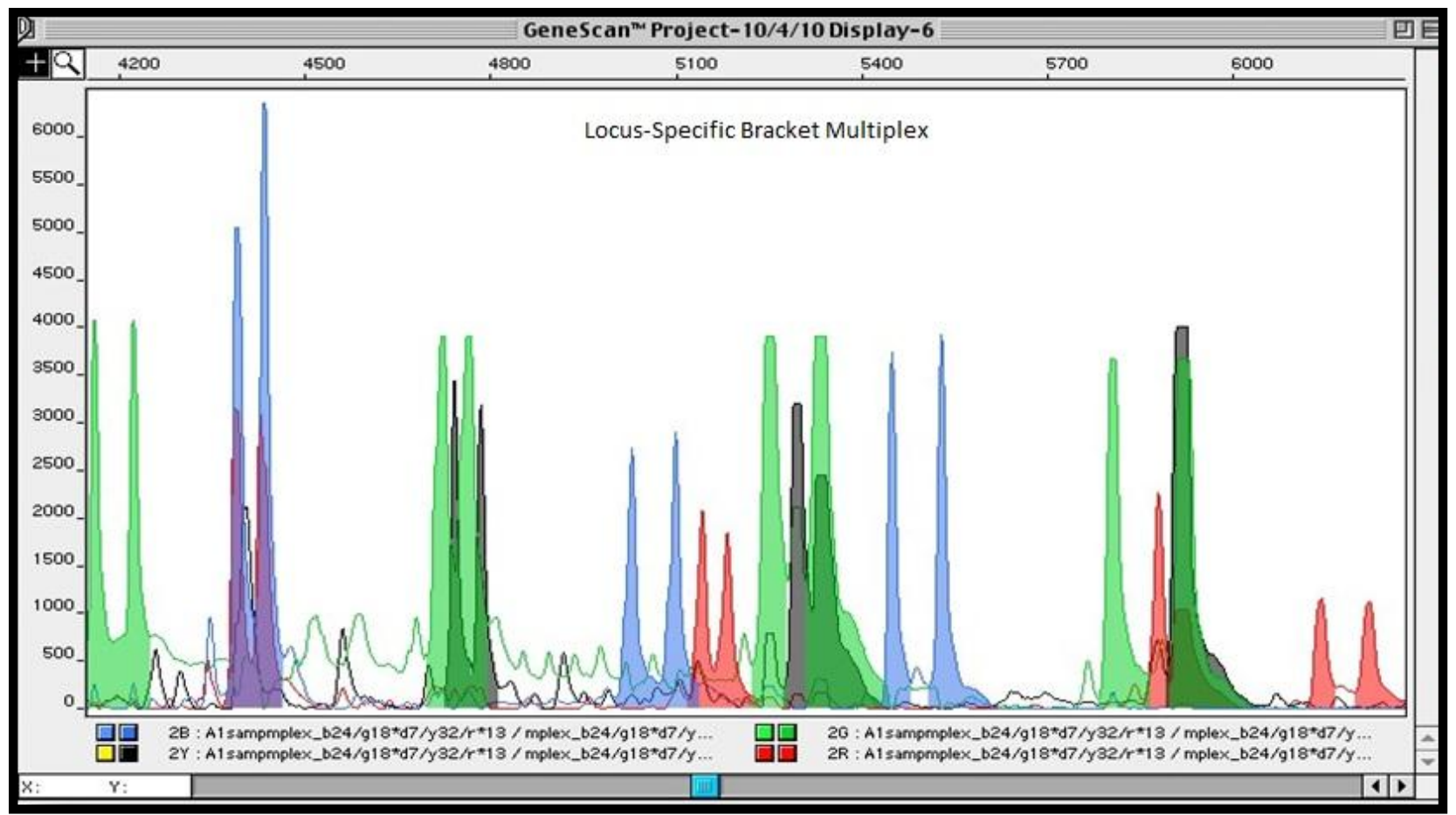

Figure 14. Optimized LSB multiplex. 


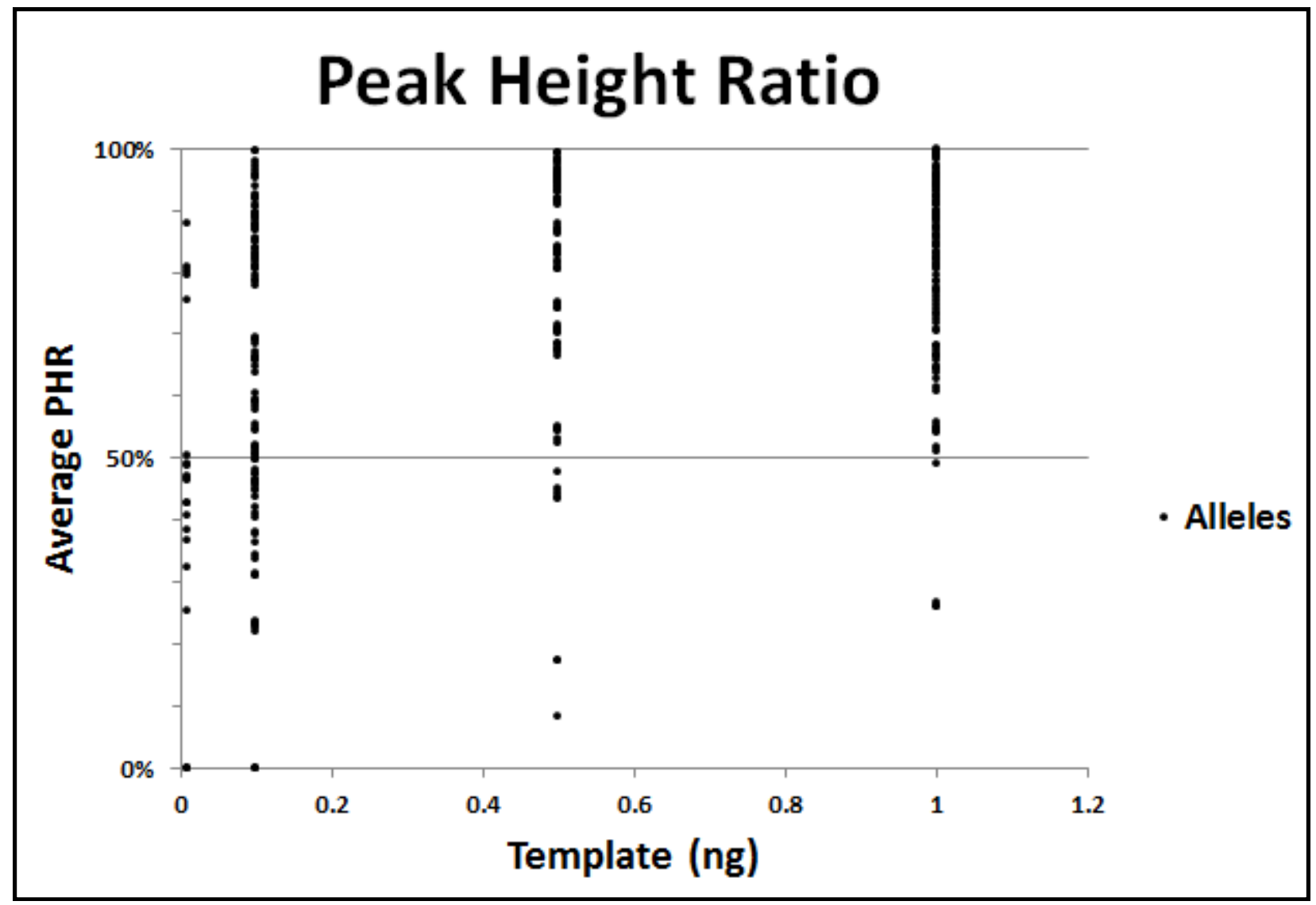

Figure 15: Average heterozygote peak height ratio for the LSB multiplex. The $X$ axis indicates thee imput DNA concentration and the y axis indicates the heterozygote peak height ratio (PHR)at each individual loci (injections were not averaged). The results illustrates 1 amplification of three separate genomic DNA extracts with the LSB multiplex for concentrations ranging from $1-0.01 \mathrm{ng}$. 


\section{Part Two: Design and synthesis of Locus Specific Brackets}




\section{Introduction}

STR allele sizing by Locus Specific Brackets requires pairs of sequence compatible DNA standards that can closely bracket a locus without overlapping with its alleles upon electrophoresis [33]. Because they are nearly identical in sequence and fragment length to alleles of the genetic loci they calibrate, all locus specific brackets can be co-electrophoresed with sample alleles in the same lane allowing for precise calibration standards [35]. Locus-Specific Brackets can be created by using an existing allele as a starting template. With the appropriate primers, the existing allele can be PCR-amplified to be shorter than the smallest common allele, and larger than the largest common allele for a particular locus. The number of repeats present in the template must be the smallest and largest available alleles for each locus. Because in order to create a bracket only a single allele needs to be present, the desired allele must be isolated when present in a heterozygous form. This can be achieved by amplifying the locus through PCR and extracting alleles from non-denaturing polyacrylamide gels (PAGE), DNA cloning. Once isolated, the allele must be amplified by PCR, separated and detected by electrophoresis. Another way of obtaining a bracket without the rigorous lab process is simply by commercially synthesizing the desired allele sequence.

\section{Isolating STR alleles through Polyacrylamide Gel Electrophoresis (PAGE)}

PAGE is a great electrophoretic technique developed to separate and purify DNA and proteins on the basis of their size, conformation, and net charge. Depending on the composition of the gel the technique can resolve DNA fragments that are short as well as in close proximity [42]. PAGE

gels have much smaller size spores ( 100-200 ̊́) than agarose gels ( 1500-2000 ̊́) allowing for higher resolution [9]. With native polyacrylamide gels, DNA fragments are separated but remain 
in their double stranded form while under denaturing conditions DNA fragments remain single stranded [43].Generally a higher level of resolution is obtained with denaturing gels given that single stranded DNA is more flexible than double stranded and interacts better with the medium, allowing closely spaced DNA fragments to be separated [44]. The disadvantage of using this method is that the process is very time consuming; the materials used for creating the gel are highly neurotoxic, back flow of DNA while loading the gels can easily cause contamination with adjacent wells, and the thin and delicate gel needs to be handle with extreme care to avoid breakage [45]

\section{Isolating STR alleles with TA DNA cloning}

DNA cloning is simply a recombinant technique that allows the transfer of a DNA fragment of interest from one organism to a self-replicating genetic element such as a bacterial plasmid [46]. Generally DNA cloning involves four basic steps. First the source and vector DNA are isolated. Second restriction enzymes cut the two DNA strands, creating ends that can connect the DNA template with the vector. The third step involves bonding the DNA template to the vector using a DNA ligase enzyme and finally the DNA is transformed into a host cell [47]. Because STR multiplex PCR products are amplified with Taq DNA polymerase TA cloning is one of the simplest methods that can be used for the purpose of allele isolation. The name TA comes from the ability of the Taq polymerase to add a single A (deoxyadenosine) to the 3' end of the PCR products, which in turn is used to directly clone the target to a linearized vector with a $3^{\prime} \mathrm{T}$ overhang [48]. A topoisomerase enzyme binds to Duplex DNA and cleaves the backbone of the DNA strand forming a covalent bond between the 3'phospahate of the cleaved strand and the topoisomerase (Figure 16). The ligation process of the PCR product causes a disruption in the 
fusion of the vector's lethal $E$-coli gene ccdB and the lacZ $\alpha$ gene permitting growth of only positive recombinants upon transformation on the competent cells [49]. Once bacterial colonies have grown the plasmid DNA can be isolated and used for sequencing or direct PCR amplification.

\section{Generating STR alleles through artificial gene synthesis}

Gene synthesis is rapidly becoming the preferred method for applications requiring the assembly of DNA sequences, both natural and engineered. These genes are normally generated by the synthesis of long oligomers, with overlapping nucleotides ( 20bp) that are later ligated and assembled into full length genes. In the same manner short tandem repeats can be synthesized and used as template for a PCR reaction. The advantage of this option is that gene synthesis services are commercially available through any major biotechnology company [50]. However a major disadvantage is that the costs are quite high. 


\section{Materials and Methods}

\section{DNA Sample preparation}

DNA samples were already extracted and quantified during the previous validation of the LSB kit as recommended by SWGDAM revised validation guidelines. Liquid blood samples (100ul) and buccal swabs were collected from 100 anonymous (WVU IRB protocol 16279). DNA extraction from the liquid blood samples was done using Qiagen (Valencia, CA) DNeasy kit according to protocol for whole nucleated blood, with a final elution of $100 \mathrm{uL}$. Buccal swab samples were extracted using the Qiagen DNeasy kit according to manufacturers' directions for DNA extraction from tissues, with a final elution of 100uL. All samples were quantified for nucleic acid content using the nanodrop spectrophotometer and further diluted to a working concentration of $1 \mathrm{ng} / \mu \mathrm{l}$. Samples were chosen depending on the number of repeats needed to make the brackets (refer to appendix section Table 4).

\section{$\underline{\text { LSB New Primers }}$}

New reverse primers were designed to amplify the targeted regions except D13S317 for which a new forward primer was designed. Primers for the short brackets were designed by deleting nucleotides of the 5 'end of the existing primer for a particular locus or by shifting the entire primer annealing site, and the primers for long brackets were design by adding nucleotides to the 5'end of the existing primer for a particular locus or by shifting the entire primer annealing site. Primers were ordered from Integrated DNA Technologies (Coralville, IA. Because all bracket specific primers would yield PCR products less than 400bp, a second set of primers was designed to increase the overall size of each PCR product to be cloned (primer sequences are 
given in Table 5 of appendix section). These primers were also designed in a way that if the primer pair failed to work together, a combination of both bracket and cloning specific primers would yield a fragment that is larger than $500 \mathrm{bp}$ and increase the success of the cloning reaction (Figure 17). The cloning specific primer pairs did not work together in all cases, but one or both primer combinations worked during the PCR reaction and only the pair that produced the larger fragment size was used to clone the brackets.

\section{$\underline{\text { PCR amplification (starting material) }}$}

All samples were amplified with $3 \mu \mathrm{L}$ of $5 \mathrm{X}$ LSB buffer (Tris $\mathrm{HCl} 67 \mathrm{Mm}, \mathrm{Ph} 8.3, \mathrm{KCl} 333 \mathrm{Mm}$, $\mathrm{MgCl}_{2} 10 \mathrm{Mm}$, gelatin $.007 \%$ ) and $3 \mu \mathrm{L}$ of $5 \mathrm{X}$ dNTPs mix (25mM each from Promega Corporation, Madison, WI). The 5U Immolase Taq polymerase from Bioline USA Inc, was diluted to $1 \mathrm{U}$ and one microliter was used per reaction. $2 \mathrm{uL}$ of each 5 pmol primer was used together with $3 \mathrm{uL}$ of water and $1 \mathrm{uL}$ of 1 ng DNA template for a total of $15 \mathrm{uL}$. All samples were amplified on a Perkin-Elmer Thermal Cycler Model 9700 model with the following cycling parameters $95^{\circ} \mathrm{C}$ for 10 minutes, then 10 cycles of $94^{\circ} \mathrm{C}$ for 30 seconds, (touchdown) $71-62{ }^{\circ} \mathrm{C}$ for 30 seconds $(30 \% \mathrm{rmp}), 72^{\circ} \mathrm{C}$ for 60 seconds $\left(20 \% \mathrm{rmp}\right.$ ), then 26 cycles of $94^{\circ} \mathrm{C}$ for 30 seconds, $59^{\circ} \mathrm{C}$ for 30 seconds $(30 \% \mathrm{rmp}), 72^{\circ} \mathrm{C}$ for 60 seconds $(20 \% \mathrm{rmp})$, and a final extension of 60 minutes at $70^{\circ} \mathrm{C}$.

\section{$\underline{\text { PCR purification }}$}

All PCR products were purified using a QIAquick PCR purification kit (Qiagen) following the manufacturer's protocol. 


\section{Direct PCR amplification of bacterial colonies}

All samples were amplified with $10 \mu \mathrm{l}$ of Promega master mix, $1 \mu 1$ of both M13 forward and reverse primer $7 \mu l$ of water and $1 \mu l$ (plasmid) or $3 \mu l$ (direct colony) template. All samples were amplified on a Perkin-Elmer Thermal Cycler Model 9700 model with the following cycling parameters $94^{\circ} \mathrm{C}$ for 10 minutes, $94^{\circ} \mathrm{C}$ for 30 seconds, $47^{\circ} \mathrm{C}$ for 30 seconds, $72^{\circ} \mathrm{C}$ for 30 seconds, and a final extension of 10 minutes at $72^{\circ} \mathrm{C}$.

\section{$\underline{\text { Polyacrylamide Gel Electrophoresis }}$}

A $12 \%$ nondenaturing gel was prepared with $21 \mathrm{ml}$ of $40 \%$ acrylamide/bis- acrylamide $(37: 1$ ratio), $500 \mu \mathrm{l}$ of $10 \%$ ammonium persulfate (APS) and $14 \mathrm{ml}$ of $5 \mathrm{X}$ Tris-Buffered Saline (TBS), and water for a total of $70 \mathrm{ml}$. The solution was degassed and then divided in half. For each $35 \mathrm{ml}$ half, $12.3 \mu$ l of tetramethylethylenediamine (TEMED) was added. $15 \mu 1$ of PCR product plus $7 \mu 1$ of PAGE 6X loading dye was loaded into the well. The gel was run for 16 hours, washed and stained with ethidium bromide (EtBr) and then visualized under UV light. A $200 \mu 1$ tip was used to make, 3 punctures in the center of the desire band and place in $20 \mu 1$ of water to soak overnight allowing the DNA to slowly diffuse into the water. The water was used the following day for PCR as a template, and amplification was confirmed using a 2\% agarose yield gel.

\section{$\underline{\text { TOPO TA Cloning }}$}

All cloning reactions were carried out using the TOPO TA cloning kit for sequencing from Invitrogen. 


\section{$\underline{\text { LB-Agar plates }}$}

All plates were prepared by adding $250 \mathrm{ml}$ of $\mathrm{dH}_{2} \mathrm{O}, 20 \mathrm{~g}$ of LB-Agar powder (VWR), mix thoroughly on a heat plate for $1 \mathrm{~min}$, autoclave the solution for $20 \mathrm{~min}$, let agar cool to $\sim 55^{\circ} \mathrm{C}$, add $50 \mu \mathrm{l}$ of $20 \mathrm{mg} / \mathrm{ml}$ of ampicillin and pour before solidification begun. Each plate was labeled and sealed appropriately. X-gal was dissolved in dimethoxy formamide $(40 \mathrm{mg} / \mathrm{ml})$ and $40 \mu 1$ were added to each at the appropriate time.

\section{Analyzing transformants through Direct PCR}

Each colony was collected with a toothpick; transfered to a reference plate for regrowth and the remaining cells were placed in $10 \mu \mathrm{l}$ of water. This was then placed in the thermal cycler for 10 minutes at $95^{\circ} \mathrm{C}, 8 \mu \mathrm{l}$ were used as template for a $\mathrm{PCR}$ reaction (see section above). Each sample was loaded onto a $2 \%$ agarose gel for identification of positive colonies.

\section{$\underline{\text { Plasmid Purification }}$}

After having grown the bacterial colonies on LB-Broth overnight, all plasmids were purified according to the instruction from Qiagen Plasmid Purification kit.

\section{$\underline{\text { Bracket mixture }}$}

Once amplified each labeled bracket was quantified and diluted to a 10ng concentration. They were then combined to specific ratios into one mixture Table 3. 


\section{Capillary electrophoresis}

$1 \mathrm{uL}$ of bracket PCR mixture and $0.5 \mathrm{uL}$ of ROX -500 size standard were combined with $15 \mathrm{uL}$ of HiDi foramide (Applied Biosystems). This mixture was denatured at $95^{\circ} \mathrm{C}$ for 3 minutes followed by snap cooling on ice for 3 minutes. Samples were then loaded onto the ABI Prism 310 Genetic analyzer (Applied Biosystems) using a 60cm capillary and POP-4 polymer (Applied Biosystems). Injection was done at $15.0 \mathrm{kV}$ for $5 \mathrm{sec}$, with a run temperature of $60^{\circ} \mathrm{C}$ and a run time of 30 minutes per sample.

\section{$\underline{\text { Artificially synthesized Brackets }}$}

Brackets to be commercially synthesized were modified (repeat numbers were added/decreased) to meet the expected size of the desired bracket.

\section{Bracket precision study}

All JOE/FAM labeled brackets data was exported from GeneScan software into Microsoft Excel and multiple injections were averaged prior to subsequent calculations. The observed sizes were subtracted from the expected and plotted against the size deviation. Both average and standard deviation were calculated for each bracket and the standard deviation was multiplied by 3 for a $99.7 \%$ confidence interval. 


\section{$\underline{\text { Results }}$}

Allele Isolation with polyacrylamide gel

Bracket isolation from a $12 \%$ non-denaturing polyacrylamide gel failed. Samples were contaminated as indicated by the extra peaks observed in the electrophoregram, and therefore were not included in the LSB mixture.

\section{Cloned Brackets}

TA-cloning successfully generated all FAM-labeled loci brackets: D13S317, TH01, and TPOX as well as JOE-labeled brackets Amelogenin short, D5S818 short, D7S820 short and long, and FGA long (Figure 18 \& 19). R0X-500 confirmed the expected sizes for each bracket. Both TAMRA \& ROX-labeled brackets were not produced due to the lack of reagents. The artificially synthesized brackets (D5S818 long and FGA short) were successfully amplified and used for genotyping in combination with the TA-cloned brackets.

\section{Brackets Precision}

The graphs in figures 20 \& 21 depict the size deviation of the newly designed brackets. All FAM labeled brackets were within the $0.5 \mathrm{bp}$ limit as expected; however, when the average and 3SD were calculated TPOX long bracket was over the $0.5 \mathrm{bp}$ limit with a 3SD equal to $0.57 \mathrm{bp}$ (Table 4). Most of the green brackets were within the $0.5 \mathrm{bp}$ limit; however with 3SD the values were greater than the $0.5 \mathrm{bp}$ for all green brackets except locus D5S818 with 3SD of $0.25 \mathrm{bp}$ and $0.23 b p$ (Table 5). 
Table 3. FAM/JOE-Bracket Mixtures concentrations

\begin{tabular}{lll}
\hline Bracket & PCR product $(\mathbf{n g})$ & Volume $(\boldsymbol{\mu l})$ \\
\hline D13S317 & 10 & 1.0 \\
Short & 10 & 1.0 \\
Long & & \\
TH01 & 10 & 1.1 \\
Short & 10 & 1.4 \\
Long & & \\
TH01 & 10 & 2.0 \\
Short & 10 & 1.6 \\
Long & & 1.9 \\
Water & & \\
& & \\
Amelogenin & 10 & 1.0 \\
Short & & \\
D5S818 & & 1.0 \\
Short & 10 & 1.0 \\
Long & 10 & \\
D7S820 & & 1.0 \\
Short & & 1.0 \\
Long & 10 & \\
FGA & 10 & 1.0 \\
Short & & 1.2 \\
Long & & 2.3 \\
water & 10 & \\
\hline
\end{tabular}


Table 4 FAM-labeled Locus Specific Brackets precision study (no sample present)

\begin{tabular}{lllllll}
\hline Brackets & D13S317-S & D13S317-L & TH01-S & TH01-L & TPOX-S & TPOX-L \\
\hline $\begin{array}{l}\text { Bracket exp. } \\
\text { (bp) }\end{array}$ & 99 & 149 & 167 & 207 & 212 & 251 \\
Mean & 99.08 & 148.94 & 167.31 & 206.71 & 211.79 & 250.76 \\
SD & 0.08 & 0.06 & 0.08 & 0.06 & 0.07 & 0.19 \\
$3 *$ SD & 0.24 & 0.18 & 0.24 & 0.18 & 0.21 & 0.57 \\
$\mathrm{n}$ & 10 & 10 & 10 & 10 & 10 & 10 \\
\hline
\end{tabular}


Table 5 JOE-labeled Locus Specific Brackets precision Study

\begin{tabular}{|c|c|c|c|c|c|c|c|}
\hline Brackets & Amelo-S & D5S818-S & D5S818-L & D7S820-S & D7S820-L & FGA-S & FGA-L \\
\hline Size (bp) & 98 & 138 & 181 & 196 & 235 & 251 & 317 \\
\hline Mean & 98.30 & 138.24 & 180.74 & 195.66 & 235.43 & 251.17 & 316.74 \\
\hline SD & 0.28 & 0.08 & 0.08 & 0.20 & 0.18 & 0.19 & 0.17 \\
\hline $3 * \mathrm{SD}$ & 0.85 & 0.25 & 0.23 & 0.61 & 0.55 & 0.58 & 0.51 \\
\hline $\mathrm{n}$ & 13 & 13 & 13 & 13 & 13 & 13 & 13 \\
\hline
\end{tabular}




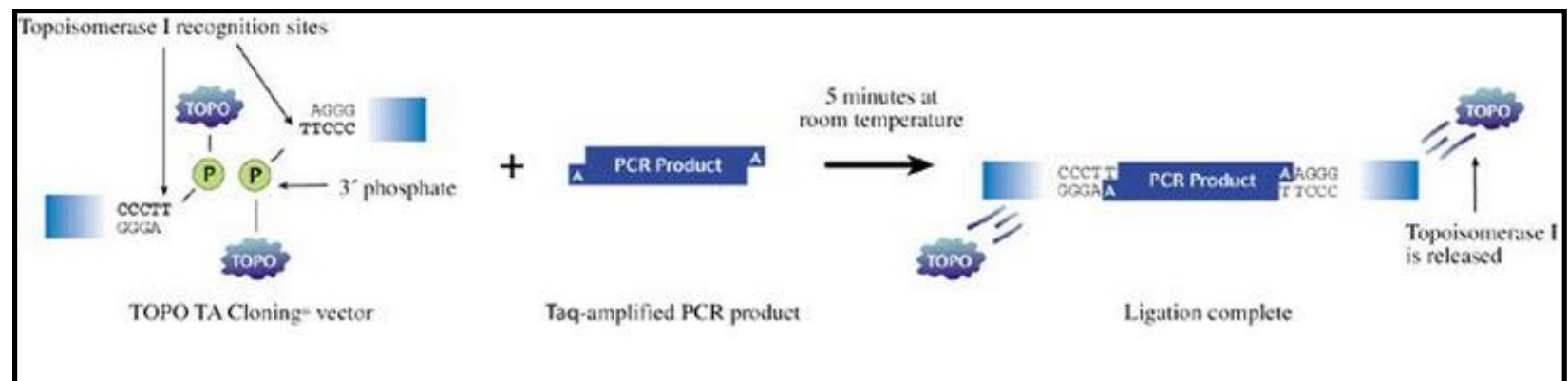

Figure 16. TOPO ${ }^{\circledR}$ TA Cloning® of Taq-amplified DNA (Available at: http://www.invitrogen.com) 


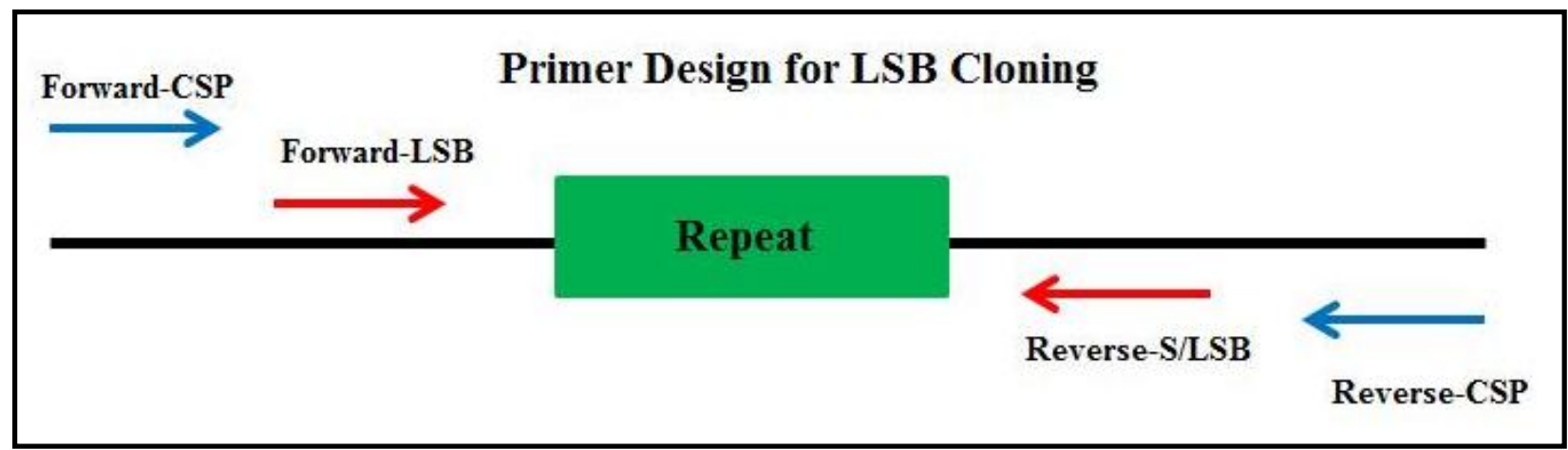

Figure 17. Illustration of primer design strategy to clone-locus specific brackets 


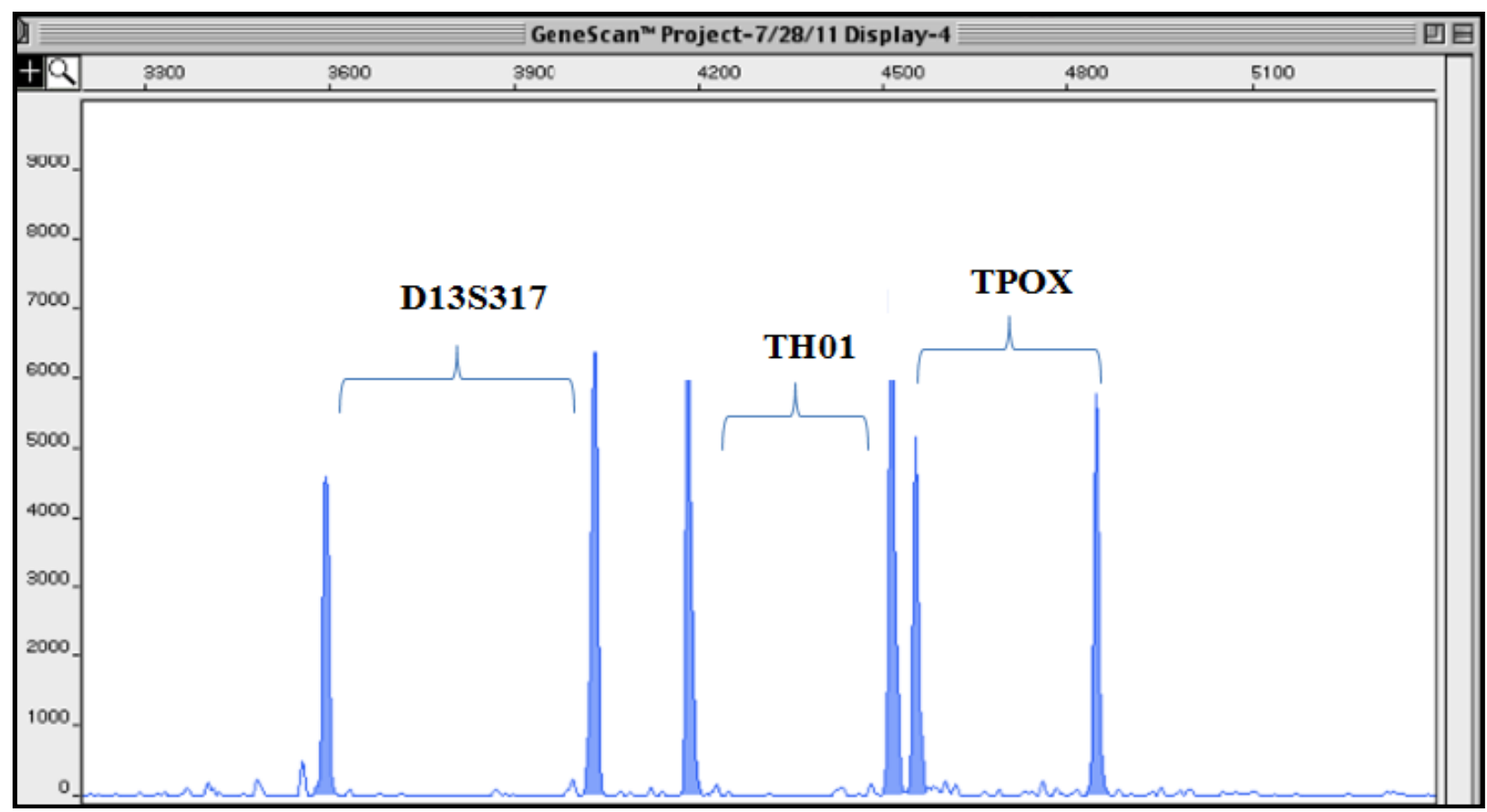

Figure 18 New FAM-labeled locus specific brackets. 


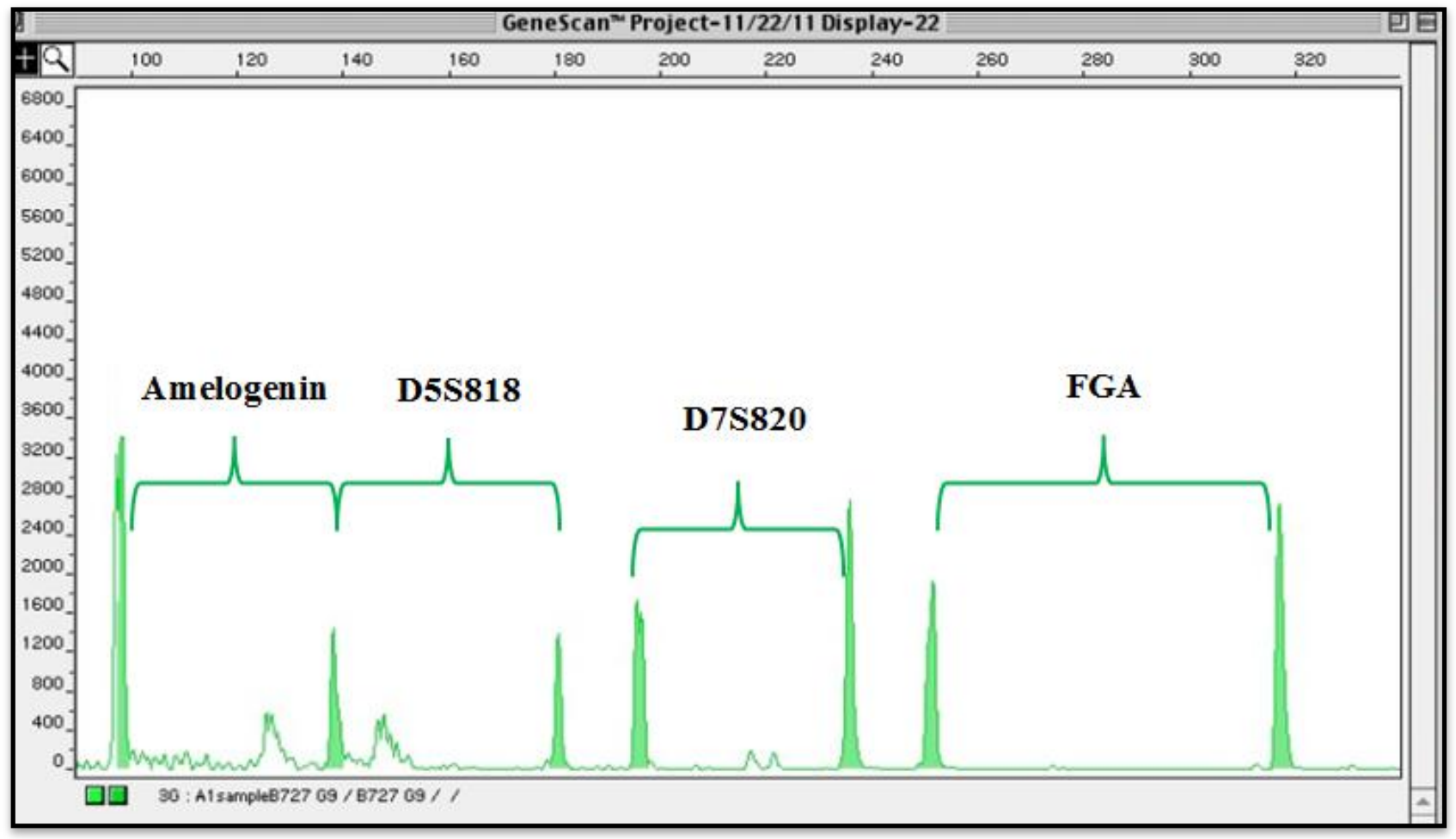

Figure 19. New JOE-labeled locus specific brackets 


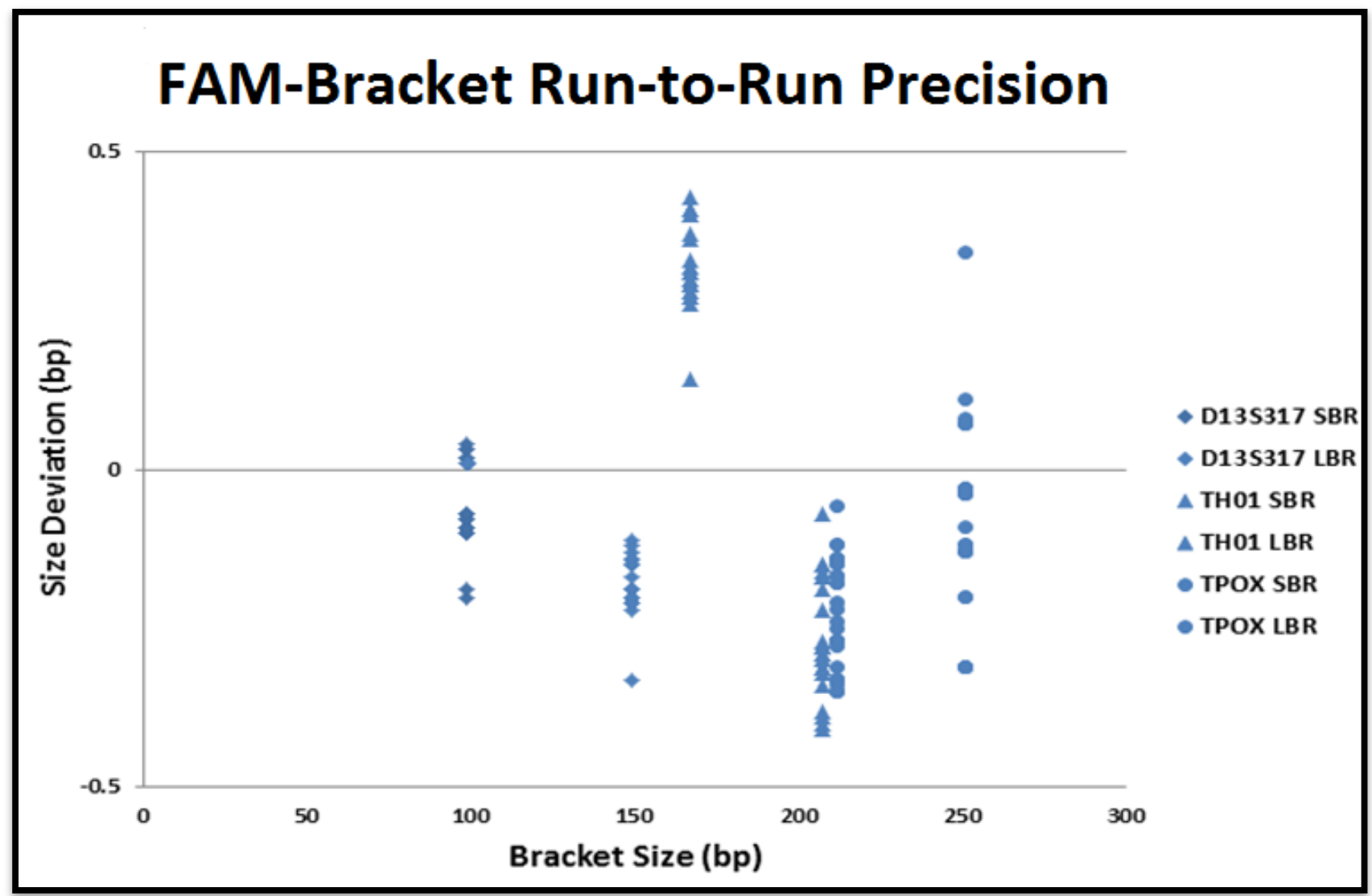

Figure 20. Size deviation for all FAM-labeled brackets 


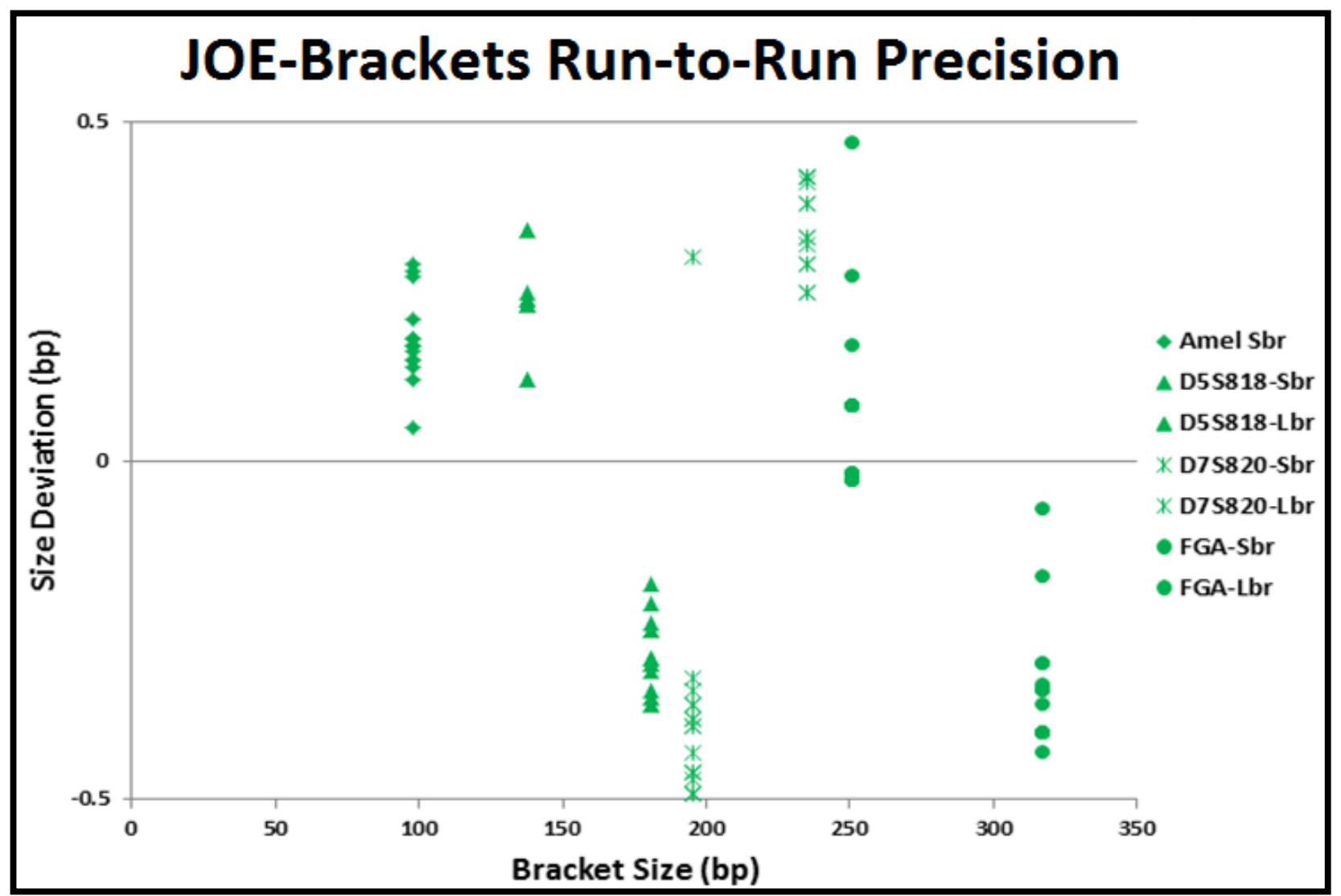

Figure 21. Size deviation for all JOE-labeled brackets 
Part Three: Preliminary evaluation of the Performance of Locus Specific Brackets as a new Human Identification Testing Kit 


\section{Introduction}

In the United States the DNA advisory Board (DAB) and the Technical Working Group on DNA Analysis Methods (TWGDAM) were established to aid the forensic community with the revision of the Quality Assurence Standard for Forensic Caseworking Laboratories and the Quality assurance Standards for DNA Databasing Laboratories [51]. Under these standards the process of validation is an important part of forensic DNA typing as it establishes the limitations and characteristics of the method and whether or not it fits the purpose for which it was developed [52]. Validation is also necessary because it demonstrates the robustness reliability and reproducibility of the method. A robust method is one in which successful results are obtained a high percentage of the time and few, if any, samples need to be repeated. A reliable method refers to one in which the obtained results are accurate and correctly reflect the sample being tested, while a reproducible method means that the same or very similar results are obtained each time a sample is tested [53]. In order to test the performance of the LSB kit two experiments were chosen to test the sensitivity and reproducibility of the kit.

\section{$\underline{\text { Reproducibility Study }}$}

A high degree of precision is needed between multiple runs in order for the procedure to be considered reliable. Precision for the separation must be $+/-0.5 b p$ to accurately distinguish between microvariant (partial repeat) alleles and complete repeats that differ by one nucleotide. Imprecision is normally expressed as the standard deviation of observed fragment size. Precision gives reproducible sizing data, precise enough to produce the expected genotype under a given set of experimental conditions [54]. 


\section{$\underline{\text { Sensitivity Study }}$}

For an STR multiplex sensitivity refers to the lowest sample DNA concentration that can be measured with confidence; also known as Limit of Detection (LOD), and the lowest concentration of DNA that can be determined in a precise and accurate manner given a set of experimental conditions [37]. Sensitivity is expressed as the lower and upper limit of DNA template concentrations that can reproducibly produce a full profile with peak heights above the analysis threshold [55]. 


\section{$\underline{\text { Materials and Methods }}$}

\section{DNA Sample preparation}

DNA samples were already extracted and quantified during the previous validation of the LSB kit as recommended by SWGDAM revised validation guidelines. Liquid blood samples (100ul) and buccal swabs were collected from 100 anonymous (WVU IRB protocol 16279). DNA extraction from the liquid blood samples was done using Qiagen (Valencia, CA) DNeasy kit according to protocol for whole nucleated blood, with a final elution of 100uL. Buccal swab samples were extracted using the Qiagen DNeasy kit according to manufacturers' directions for DNA extraction from tissues, with a final elution of $100 \mathrm{uL}$. All samples were quantified for nucleic acid content using the nanodrop spectrophotometer.

\section{$\underline{\text { PCR amplification }}$}

All samples were amplified with $3 \mu \mathrm{L}$ of $5 \mathrm{X}$ LSB buffer (Tris $\mathrm{HCl} 67 \mathrm{Mm}, \mathrm{Ph} 8.3, \mathrm{KCl} 333 \mathrm{Mm}$, $\mathrm{MgCl} 210 \mathrm{Mm}$, gelatin $.007 \%)$ and $3 \mu \mathrm{L}$ of $5 \mathrm{X}$ dNTPs mix $(25 \mathrm{mM}$ each from Promega Corporation, Madison, WI). The 5U Immolase Taq polymerase from Bioline USA Inc, was diluted to $1 \mathrm{U}$ and one microliter was used per reaction. $2 \mathrm{uL}$ of each $5 \mathrm{pmol}$ primer was used together with $3 \mathrm{uL}$ of water and $1 \mathrm{uL}$ of $1 \mathrm{ng}$ DNA template for a total of $15 \mathrm{uL}$. All samples were amplified on a Perkin-Elmer Thermal Cycler Model 9700 model with the following cycling parameters $95^{\circ} \mathrm{C}$ for 10 minutes, then 10 cycles of $94^{\circ} \mathrm{C}$ for 30 seconds, (touchdown) $71-62{ }^{\circ} \mathrm{C}$ for 30 seconds $(30 \% \mathrm{rmp}), 72^{\circ} \mathrm{C}$ for 60 seconds ( $20 \% \mathrm{rmp}$ ), then 26 cycles of $94^{\circ} \mathrm{C}$ for 30 seconds, $59^{\circ} \mathrm{C}$ for 30 seconds ( $\left.30 \% \mathrm{rmp}\right), 72^{\circ} \mathrm{C}$ for 60 seconds $(20 \% \mathrm{rmp}$ ), and a final extension of 60 minutes at $70^{\circ} \mathrm{C}$. 


\section{Capillary Electrophoresis}

1uL of PCR product, 0.5UL of ROX-500, and 1ul of JOE/FAM LSB brackets mixture were combined with a $15 \mu \mathrm{l}$ of HiDi foramide (Applied Biosystems). This mixture was denatured at $95^{\circ} \mathrm{C}$ for 3 minutes followed by snap cooling on ice for 3 minutes. Samples were then loaded

onto the ABI Prism 310 Genetic analyzer (Applied Biosystems) using a 60cm capillary and POP4 polymer (Applied Biosystems). Injection was done at $15.0 \mathrm{kV}$ for $5 \mathrm{sec}$, with a run temperature of $60^{\circ} \mathrm{C}$ and a run time of 30 minutes per sample.

\section{$\underline{\text { Data Analysis }}$}

\section{$\underline{\text { Sensitivity }}$}

Three samples were tested; each at DNA template concentrations of $1 \mathrm{ng}, 0.5 \mathrm{ng}, 0.1 \mathrm{ng}$, and $0.01 \mathrm{ng}$ for a total of 12 samples and all 12 samples were run on the genetic analyzer on three separate days. The data were exported from GeneScan software into Microsoft Excel and peak height values from all 3 injections were averaged prior to subsequent calculations. Intralocus balance or heterozygous peak height ratio was calculated by dividing the height of the smallest allele by the height of the largest allele at each locus and then expressed as a percentage. Intracolor was evaluated by first averaging all heterozygote peak heights and then dividing homozygous peak height in half. Once normalized for diploidy, the lowest allele height was divided by the largest allele height labeled with a given dye and then expressed as a percentage. Intercolor balance was calculated in a similar manner to intracolor, except the comparison was made across all loci regardless of the dye color [56]. The average of all peak heights were also calculated per locus, based on all three samples and three separate injections. 


\section{$\underline{\text { Precision }}$}

Data from GeneScan software were imported into Microsoft Excel. The observed allele sizes were subtracted from their expected values. The size deviation was plotted against their expected size. Their mean and standard deviations were calculated. TAMRA \& ROX-labeled loci were not analyzed for this study because their brackets are yet to be developed.

\section{Genotype concordance}

Nine samples amplified with the FAM labeled multiplex and 3 samples for the JOE labeled multiplex were used for genotyping and their allele calls were compared with the genotypes obtained from the Identifiler kit. Using Microsoft Excel simple genotyping software was written to automatically generate allele calls based on comparison to a ROX internal size standard. The software simply subtracts the conserved region length (primer binding site to the edge of repeat) from the total fragment length, the reminder is divided by 4 and the results are assigned as the allele call. For partial repeats the software was simply modified to convert repeats with decimal point of $.25, .5 .75$ to $.1, .2$, and .3 allele calls (e.g. an allele call of 9.75 repeat is converted into 9.3). 


\section{$\underline{\text { Results }}$}

\section{LSB Multiplex Sensitivity}

For three separate genomic DNA sources with input ranges from $0.01 \mathrm{ng}-1 \mathrm{ng}$ all but one sample (BKA, 0.5ng) were successfully amplified (Figure 22) with the highest analyzed peak at 7945 RFUs for 1ng amplification at locus TH01 and the lowest analyzed peak was observed in locus D8S1179 at 362 RFUs also at 1ng of DNA concentration. Peak heights decreased with decreasing DNA concentration. At $0.5 \mathrm{ng}$ of DNA the highest RFU was observed at locus D7S820 with a score of 7098 RFUs and the lowest at locus D16S539 with a score of 258RFUs. At $0.1 \mathrm{ng}$, the highest score was 5417 RFUs at locus TH01 and the lowest was D8S1179 with a score of 380RFUs, while one sample experienced allele dropout in locus TPOX. All alleles average heights were calculated based on 3 separate injections for concentrations of 1 ng and 0.1 ng and based on two injections for 0.5ng (Figure23).

At 1 ng the peak height ratio for most alleles were above $60 \%$ except 2 outliers, one at $26.1 \%$ (TPOX sample B910) and the other with 50.3\% (D8S1179 sample BKA). At 0.5ng 4 outliers were observed with high peak imbalance, TPOX sample B910 (13\%), D5S818 sample B910 with 44\%, D8S1179 for both samples B910 and B483 with 54\%. Greater locus imbalance was observed with 0.1ng with one allele dropout at TPOX for sample B910 while most loci experienced allele dropout at 0.01ng (Figure 24).

With 1ng of DNA input and 3SD the average heterozygous peak ratio for TH01, D7S820, FGA, vWA, D21S11, D18S51, D3S1358, and D16S539 were observed above 60\% as they should be. However; D5S818 and D13S313 were observed slightly higher than 46\%, while D8S1179 and 
CSF1PO were at $37 \%$ and $39 \%$ respectively Table 6. Due to the high allele imbalance at locus TPOX the average PHR was difficult to accurately be calculated and Intracolor balance (expected at around 50\%) was not obtained at the expected level. The closest to this balance was $49 \%$ by TAMRA-labeled at $1 \mathrm{ng}, 46 \%$ and $0.5 \mathrm{ng}$. Only a $7 \%$ intercolor balance was observed at $1 \mathrm{ng}, 10 \%$ at $0.5 \mathrm{ng}$ and was not accurately calculated at $0.1 \mathrm{ng}$ due the presence of allele dropout in locus TPOX Table 7. The same applies for $0.01 \mathrm{ng}$ were most alleles failed to be successfully amplified.

\section{Precision test}

Based on nine samples and three injections, a total of 150 measurements were obtained for all FAM-Labeled alleles. At locus D13S317 53/54 alleles were within the 0.5bp limit, as well as all 39/39 for locus TH01 and 35/39 for TPOX. All 78 JOE-labeled allele were also measured. For locus D5S818 23/25 fell within the expected range, 28/29 for locus D7S820 and 22/24 for locus FGA Figures $25 \&$ 26. For amelogenin marker 13/16 measured fragments were also within the expected range. Based on 3SDs the majority of FAM-labeled alleles were well within the expected 0.5 bp limit except TH01 allele 6, and TPOX allele 11 see Table 8. For JOE labeled loci FGA allele 19 was over $0.5 \mathrm{bp}$ limit with 3 SD of $0.72 \mathrm{bp}$ Table 9.

\section{Genotype concordance}

A total of 228 comparisons were made for loci D13S317, TH01, TPOX, D5S8181, D7S820, FGA, and amelogenin between the LSB kit and the commercial kit identifiler. Full concordance was observed at locus D13S317 and TH01 were all 58/58 genotype comparisons were correct. Locus TPOX was the only one affected at alleles 8 and 11 were systematically called $8.1 \& 11.1$ 
(these sizes were also 1bp higher than expected) for all three injections in 3 samples; however in two occasion two samples were genotyped correctly during one injection but incorrectly in the others for a total of $36 / 58$ correct genotypes (sizes match the incorrect genotype in both samples). For JOE-labeled loci, a total of 77 genotype comparisons were made. 50/72 genotypes were correct while 22 were incorrect. The discrepancies were observed at locus amelogenin where 2 out of the three samples had both X \& Y fragments "off ladder" on two out of the three injections. Locus D5S818 had 2 samples were allele 12, was consistently called 12.1 in all three injections. Locus D7S820 alleles 11 and12 were genotyped as $11.1 \& 12.1$ in one sample and locus FGA, two samples repeatedly called alleles $18 \& 19$ as 18.1 and 19.1 respectively; In all of these cases, the fragment sizes were also off by $1 \mathrm{bp}$. See Table $10 \& 11$. 
Table 6Average peak Height Ratio after all three sample injections were averaged

\begin{tabular}{llll}
\hline $\begin{array}{l}\text { STR } \\
\text { Locus }\end{array}$ & $\begin{array}{l}\text { Average } \\
\text { PHR }\end{array}$ & SD & 3SD \\
\hline D13S317 & $76 \%$ & $10 \%$ & $46 \%$ \\
TH01 & $96 \%$ & $2 \%$ & $91 \%$ \\
TPOX & N/A & N/A & N/A \\
Amel & $93 \%$ & $7 \%$ & $70 \%$ \\
D5S818 & $82 \%$ & $11 \%$ & $50 \%$ \\
D7S820 & $96 \%$ & $3 \%$ & $87 \%$ \\
FGA & $82 \%$ & $4 \%$ & $69 \%$ \\
vWA & $88 \%$ & $8 \%$ & $65 \%$ \\
D21S11 & $89 \%$ & $4 \%$ & $78 \%$ \\
D18S51 & $80 \%$ & $2 \%$ & $75 \%$ \\
D3S1358 & $93 \%$ & $6 \%$ & $75 \%$ \\
D8S1179 & $58 \%$ & $7 \%$ & $37 \%$ \\
D16S539 & $83 \%$ & $6 \%$ & $64 \%$ \\
CSF1PO & $77 \%$ & $13 \%$ & $39 \%$ \\
\hline
\end{tabular}


Table 7. Intra locus \& intracolor balance for all STR loci at different DNA concentrations (all three injections were averaged per sample)

\begin{tabular}{llll}
\hline Loci & $\begin{array}{l}\text { Template } \\
\text { 1ng }\end{array}$ & $\begin{array}{l}\text { Template } \\
\text { 0.5ng }\end{array}$ & $\begin{array}{l}\text { Template } \\
\text { 0.1ng }\end{array}$ \\
\hline D13S317 & $31 \%$ & $66 \%$ & $51 \%$ \\
TH01 & $94 \%$ & $77 \%$ & $55 \%$ \\
TPOX & $26 \%$ & $13 \%$ & dropped \\
Amel & $48 \%$ & $49 \%$ & $15 \%$ \\
D5S818 & $42 \%$ & $82 \%$ & $30 \%$ \\
D7S820 & $77 \%$ & $79 \%$ & $31 \%$ \\
FGA & $77 \%$ & $87 \%$ & $47 \%$ \\
vWA & $62 \%$ & $70 \%$ & $41 \%$ \\
D21S11 & $58 \%$ & $68 \%$ & $23 \%$ \\
D18S51 & $65 \%$ & $51 \%$ & $50 \%$ \\
D3S1358 & $47 \%$ & $70 \%$ & $46 \%$ \\
D8S1179 & $46 \%$ & $54 \%$ & $28 \%$ \\
D16S539 & $68 \%$ & $58 \%$ & $45 \%$ \\
CSF1PO & $65 \%$ & $85 \%$ & $21 \%$ \\
\hline Intracolor & & \\
\hline FAM & $20 \%$ & $10 \%$ & $11 \%$ \\
JOE & $30 \%$ & $22 \%$ & $20 \%$ \\
TAMRA & $49 \%$ & $46 \%$ & $23 \%$ \\
ROX & $7 \%$ & $12 \%$ & $10 \%$ \\
\hline
\end{tabular}


Table 8 Average sizes for FAM-labeled loci. All allele sizes are based on ROX-500 size standard and sized by GeneScan Software.

\begin{tabular}{lrrrrr}
\hline D13S317 & $\mathbf{8}$ & $\mathbf{9}$ & $\mathbf{1 1}$ & $\mathbf{1 2}$ & $\mathbf{1 3}$ \\
\hline Mean & 111.99 & 116.00 & 123.95 & 128.09 & 132.17 \\
SD & 0.08 & 0.05 & 0.09 & 0.08 & 0.06 \\
$\mathbf{3}$ SD & 0.23 & 0.15 & 0.26 & 0.23 & 0.19 \\
Exp & 112 & 116 & 124 & 128 & 132 \\
$\mathbf{n}$ & 15 & 3 & 20 & 9 & 6 \\
\hline TH01 & $\mathbf{6}$ & $\mathbf{7}$ & $\mathbf{8}$ & $\mathbf{9}$ & $\mathbf{9 . 3}$ \\
Mean & 179.16 & 183.15 & 187.03 & 190.97 & 193.83 \\
SD & 0.30 & 0.11 & 0.07 & 0.06 & 0.07 \\
$\mathbf{3 * S D}$ & 0.90 & 0.34 & 0.20 & 0.18 & 0.22 \\
Exp & 179 & 183 & 187 & 191 & 194 \\
$\mathbf{n}$ & 18 & 12 & 9 & 12 & 6 \\
\hline TPOX & $\mathbf{8}$ & $\mathbf{9}$ & $\mathbf{1 0}$ & $\mathbf{1 1}$ & \\
Mean & 226.26 & 230.60 & 234.86 & 239.29 & 0.22 \\
SD & 0.07 & 0.10 & 0.11 & 0.67 & \\
$\mathbf{3 * S D}$ & 0.22 & 0.30 & 0.32 & 239 & \\
Exp & 226 & 231 & 235 & 3 & \\
$\mathbf{n}$ & 12 & 9 & 3 & & \\
\hline
\end{tabular}


Table 9. Average sizes for JOE-labeled loci. All allele sizes are based on ROX-500 size standard and sized by GeneScan software.

\begin{tabular}{|c|c|c|c|c|c|c|}
\hline Amelogenin & $X$ & $\mathrm{Y}$ & & & & \\
\hline Mean & 103.12 & 108.44 & & & & \\
\hline SD & 0.58 & 0.05 & & & & \\
\hline $3 *$ SD & 1.75 & 0.16 & & & & \\
\hline $\exp$ & 103 & 108 & & & & \\
\hline n & 9 & 6 & & & & \\
\hline D5S818 & 11 & 12 & 13 & & & \\
\hline Mean & 156.13 & 160.42 & 164.44 & & & \\
\hline SD & 0.07 & 0.08 & 0.04 & & & \\
\hline $3 * \mathrm{SD}$ & 0.22 & 0.23 & 0.13 & & & \\
\hline Exp & 156 & 160 & 164 & & & \\
\hline $\mathbf{n}$ & 11 & 9 & 5 & & & \\
\hline D7S820 & 8 & 10 & 11 & & & \\
\hline Mean & 208.21 & 216.92 & 221.37 & & & \\
\hline SD & 0.06 & 0.09 & 0.21 & & & \\
\hline $3 * \mathrm{SD}$ & 0.18 & 0.27 & 0.62 & & & \\
\hline Exp & 208 & 217 & 221 & & & \\
\hline n & 7 & 12 & 5 & & & \\
\hline$\overline{\text { FGA }}$ & 18 & 19 & 21 & 22 & 23 & 24 \\
\hline Mean & 261.83 & 265.79 & 273.24 & 277.12 & 280.86 & 284.62 \\
\hline SD & 0.11 & 0.24 & 0.09 & 0.19 & 0.12 & 0.08 \\
\hline $3 * \mathrm{SD}$ & 0.32 & 0.72 & 0.27 & 0.57 & 0.35 & 0.24 \\
\hline Exp & 262 & 266 & 273 & 277 & 281 & 285 \\
\hline $\mathbf{n}$ & 3 & 4 & 4 & 5 & 5 & 3 \\
\hline
\end{tabular}


Table 10. Genotype concordance test between the LSB kit and Indentifiler kit for all FAMlabeled loci.

\begin{tabular}{lllll}
\hline Sample & D13S317 M1 & D13S317 M2 & D13S317 M3 & Identifiler \\
\hline B551 & 11,13 & 11,13 & 11,13 & 11,13 \\
S972 & 8,12 & 8,12 & 8,12 & 8,12 \\
B276 & 11,12 & 11,12 & 11,12 & 11,12 \\
B985 & 8,11 & 8,11 & 8,11 & 8,11 \\
S999 & 8,11 & 8,11 & 8,11 & 8,11 \\
B581 & 8,11 & 8,11 & 8,11 & 8,11 \\
B188 & 12,12 & 12,12 & 12,12 & 12,12 \\
S800 & 8,11 & 8,11 & 8,11 & 8,11 \\
B142 & 9,13 & 9,13 & 9,13 & 9,13 \\
\hline & TH01M1 & TH01 M2 & TH01 M3 & \\
\hline B551 & 6,7 & 6,7 & 6,7 & 6,7 \\
S972 & 9,9 & 9,9 & 9,9 & 9,9 \\
B276 & 6,8 & 6,8 & 6,8 & 6,8 \\
B985 & $6,9.3$ & $6,9.3$ & $6,9.3$ & $6,9.3$ \\
S999 & $6,9.3$ & $6,9.3$ & $6,9.3$ & $6,9.3$ \\
B581 & 7,8 & 7,8 & 7,8 & 7,8 \\
B188 & 6,7 & 6,7 & 6,7 & 6,7 \\
S800 & 6,9 & 6,9 & 6,9 & 6,9 \\
B142 & 7,9 & 7,9 & 7,9 & 7,9 \\
\hline & TPOX M1 & TPOX M2 & TPOX M3 & \\
\hline B551 & 11,11 & $11.1,11.1$ & $10.3,10.3$ & 11,11 \\
S972 & 11,11 & 11,11 & 11,11 & 11,11 \\
B276 & $7.3,9$ & $7.3,9$ & $7.3,9$ & 8,7 \\
B985 & 10,11 & 10,11 & 10,11 & 10,11 \\
S999 & $9,11.1$ & 9,11 & 9,11 & 9,11 \\
B581 & $7.3,7.3$ & $7.3,7.3$ & $7.3,7.3$ & 8,8 \\
B188 & 9,9 & 9,9 & 9,9 & 9,9 \\
S800 & 11,11 & 11,11 & 11,11 & 11,11 \\
B142 & 8,8 & $7.3,7.3$ & $7.3,7.3$ & 8,8 \\
\hline & & & & \\
\hline
\end{tabular}


Table 11. Genotype concordance test between the LSB kit and Indentifiler kit for all JOElabeled loci.

\begin{tabular}{lllll}
\hline Sample & Amelogenin M1 & Amelogenin M2 & Amelogenin M3 & Identifiler \\
\hline B483 & $X, Y$ & $X, Y$ & $X, Y$ & $X, Y$ \\
B910 & OL,OL & $X, X$ & X,X & X,X \\
BKA & X,Y & $X, Y$ & X,OL & X,Y \\
\hline & D5S818 & D5S818 & D5S818 & \\
\hline & & & & \\
B483 & 11,12 & 11,12 & $11,12.1$ & 11,12 \\
B910 & 11,13 & 11,13 & 11,13 & 11,13 \\
BKA & 11,12 & 11,12 & 11,12 & 11,12 \\
\hline & D7S820 & D7S820 & D7S820 & \\
\hline B483 & 8,10 & 8,10 & $8,10.1$ & 8,10 \\
B910 & $10.1,11.1$ & $10.1,11.1$ & $10.1,11.1$ & 10,11 \\
BKA & $8,10.1$ & $8,10.1$ & $8,10.1$ & 8,10 \\
\hline & FGA & FGA & FGA & \\
\hline B483 & 23,24 & 23,24 & 23,24 & 23,24 \\
B910 & $18.1,21$ & $18.1,21$ & $18.1,21$ & 18,21 \\
BKA & $19.1,22$ & $19.1,22$ & $19.1,22$ & 19,22 \\
\hline
\end{tabular}




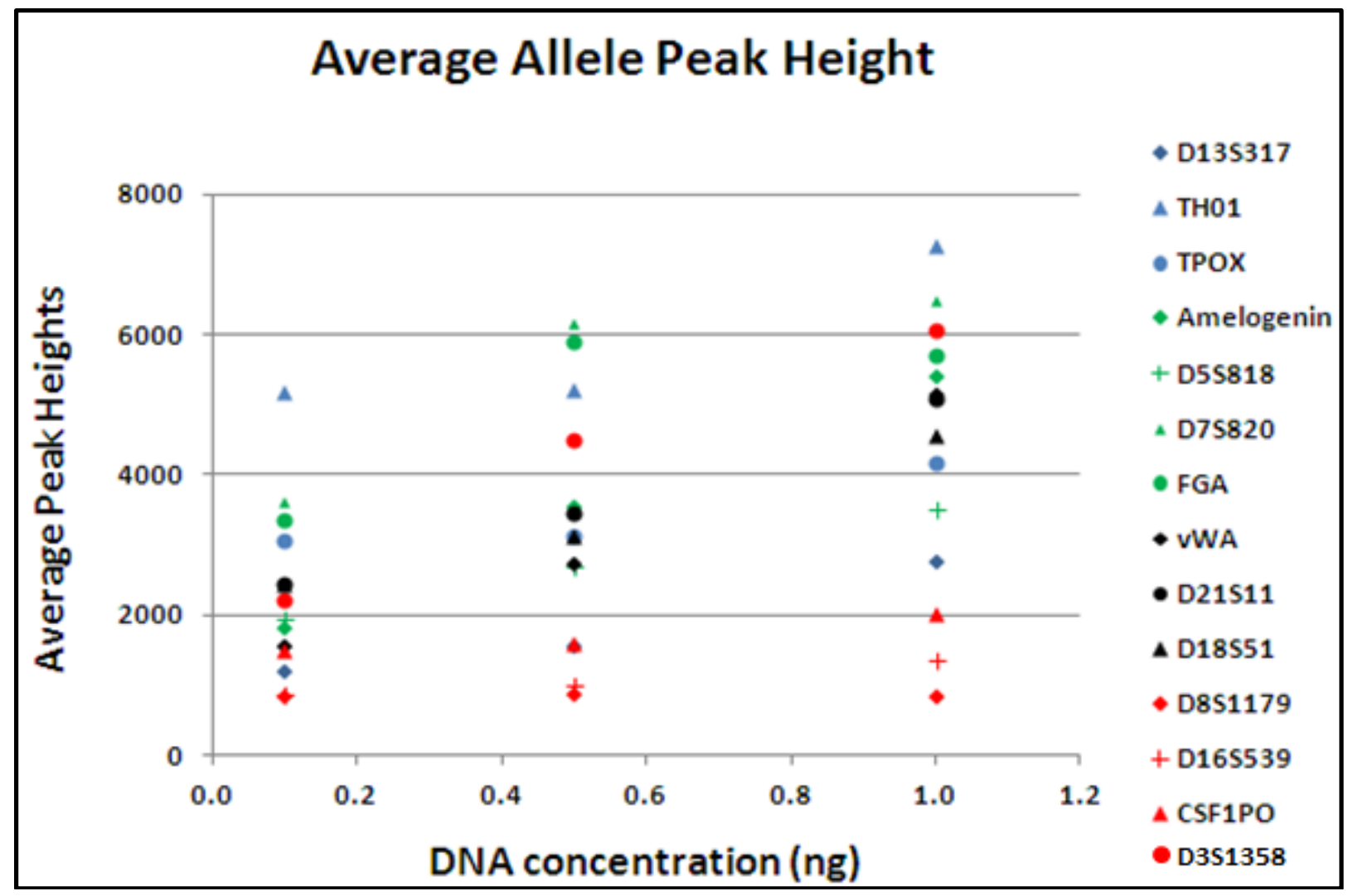

Figure 22. LSBs allele average peak heights. Based on 3 separate injections from three distinct samples sources at DNA concentrations of 1, 0.5, and 0.1(0.01ng not included due to failure to obtain full profiles). 


\section{Average Heterozygote Allele PHR Ratio}

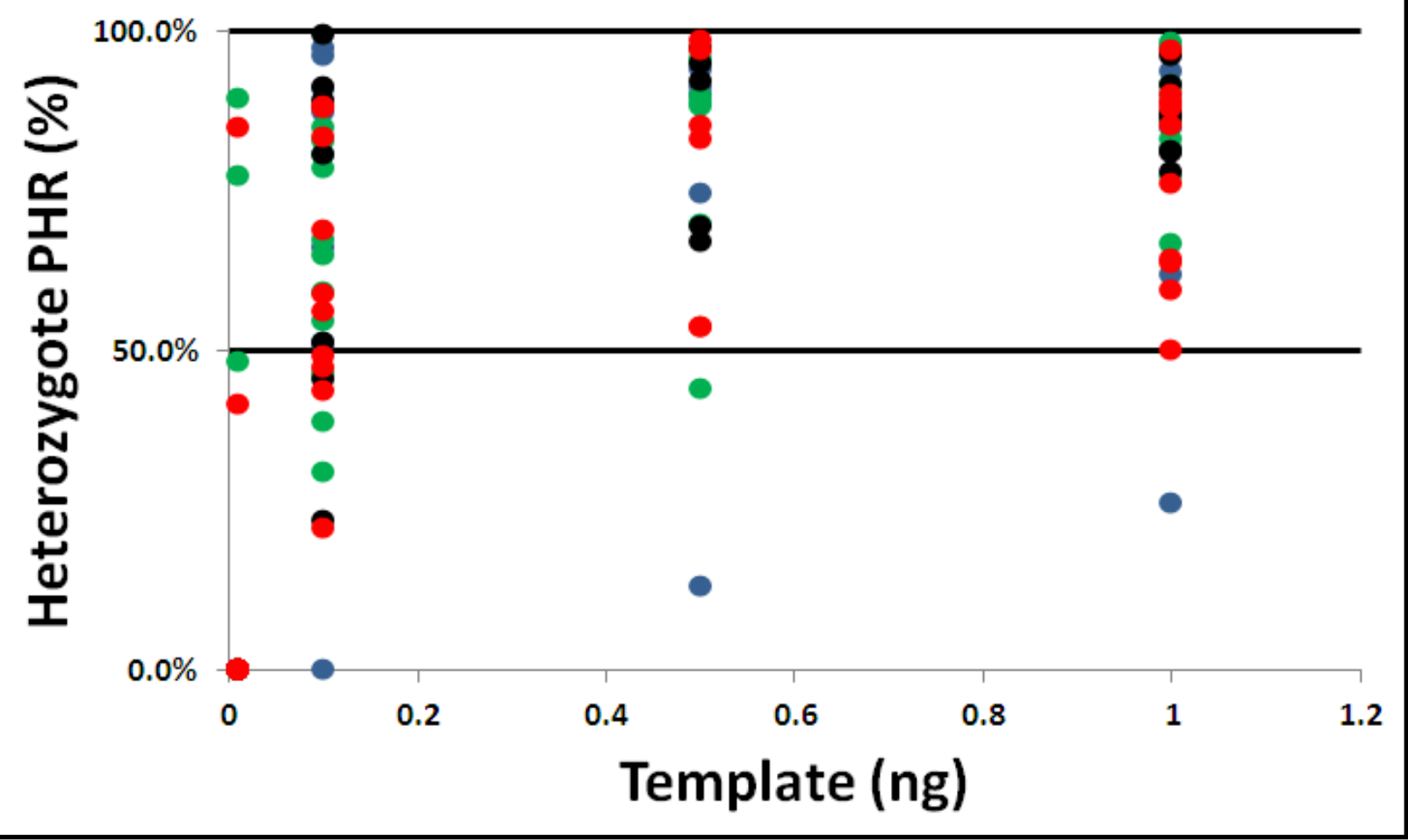

Figure 173. Heterozygous peak height ratios with various imputs of DNA concentratios. The $\mathrm{x}$ axis indicates thee imput DNA concentration and the $\mathrm{y}$ axis indicates the heterozygote peak height ratio (PHR)at each individual loci (multiple injections were avereaged). The results illustrates 1 amplification of three separate genomic DNA with the LSB multiplex for concentrations of $1,0.5$, and 0.1 , and $0.01 \mathrm{ng}$. 


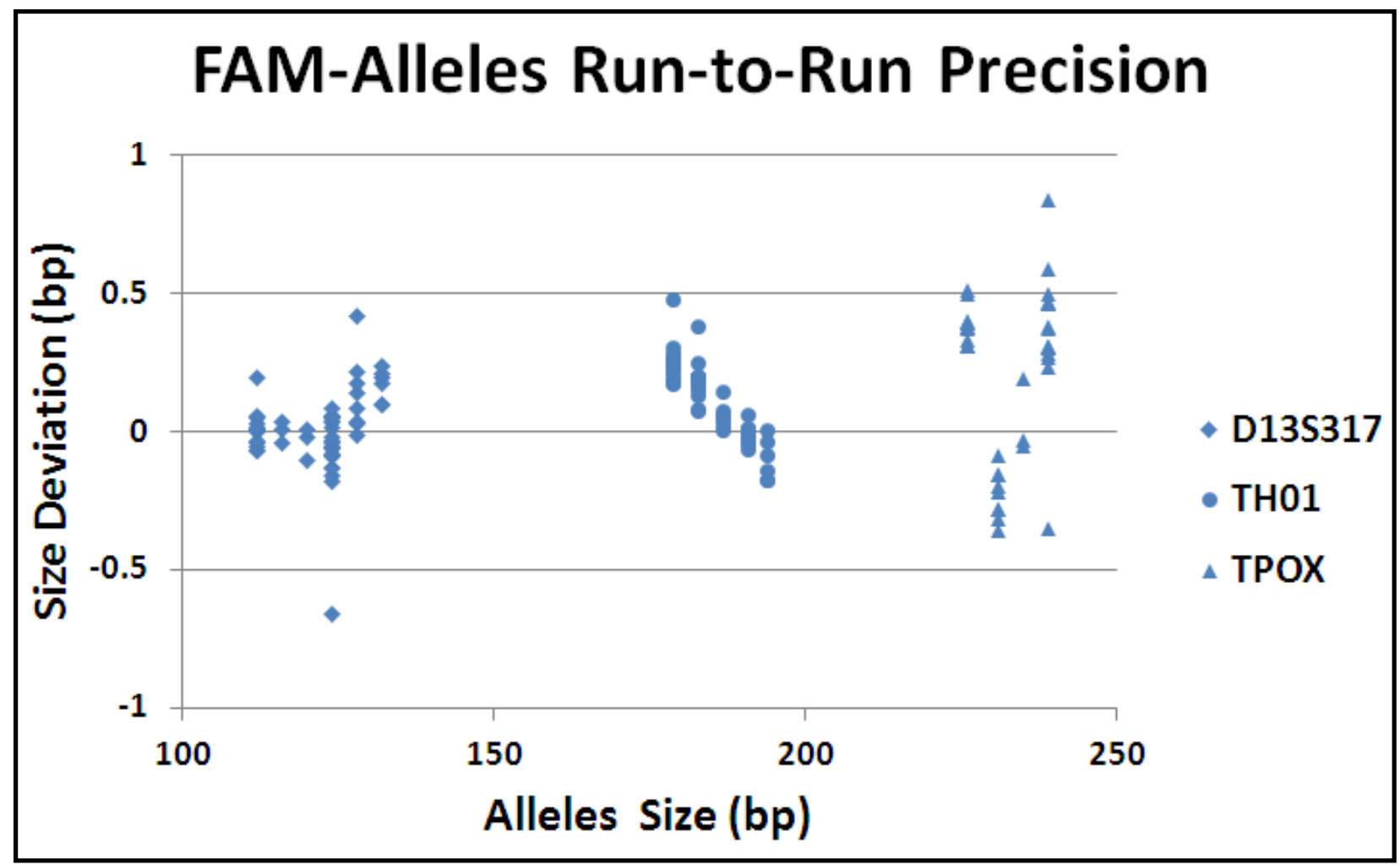

Figure 24. Size deviation for all FAM-labeled alleles based on sizes generated by ROX internal size standard. All data points are based on 9 samples with three separate injections. 


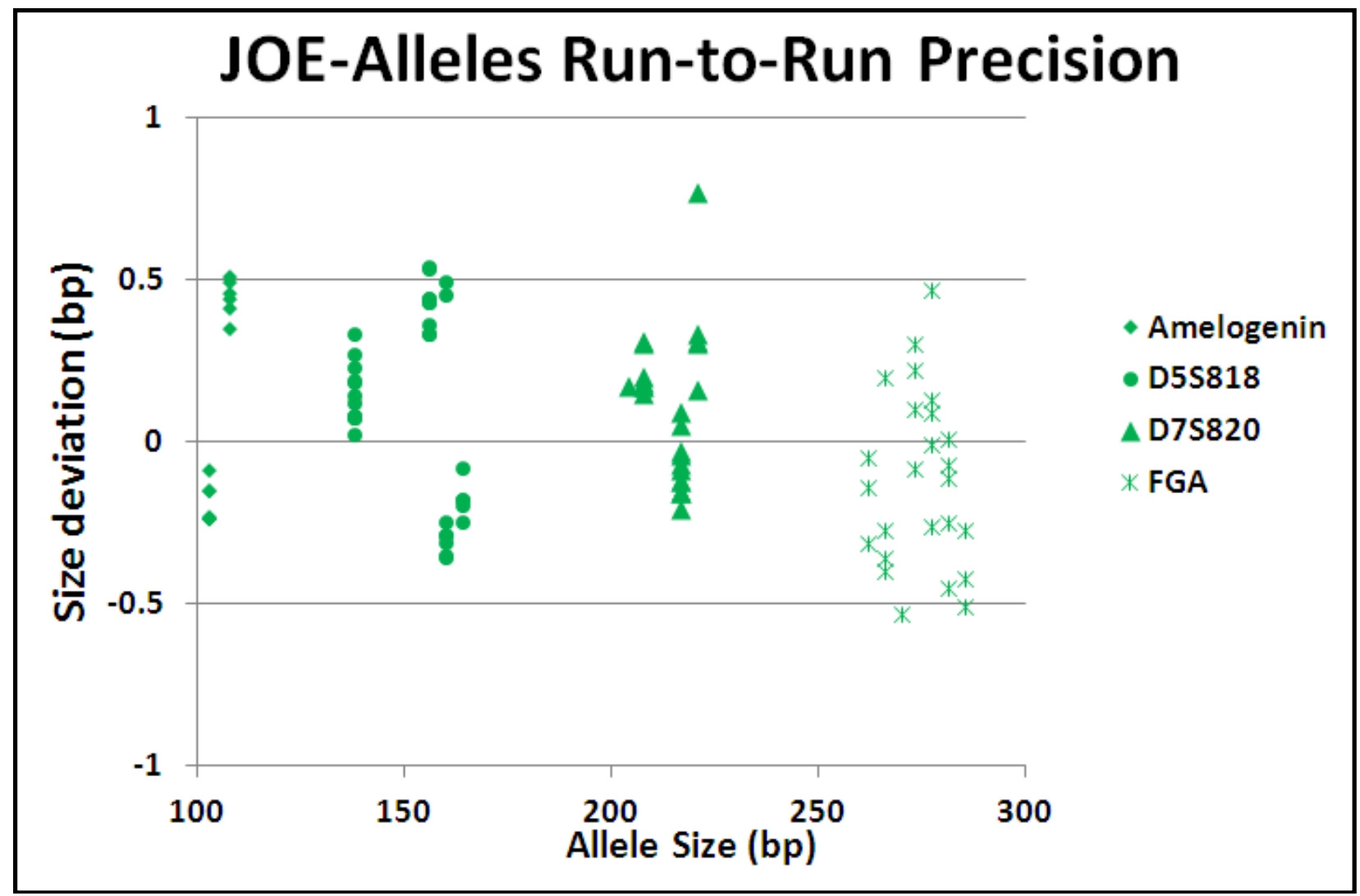

Figure 25. Size deviation for all JOE-labeled alleles based on sizes generated by ROX

internal size standard. All data points are based on 3 samples with three separate injections and three samples that were only injected once. 


\section{Discussion \& Future Research}

The LSB STR multiplex kit correctly typed $81 \%$ of all alleles using 1ng of DNA concentration from a single source, and in all three samples tested, full profiles were recovered, with allele heights well above the analysis threshold. Although full profiles were obtained, the low heterozygous PHR observed together with the high level of stutter may cause problems during future mixture studies. The LSB kit also showed that $87 \%$ of all sample alleles were accurate sized; by the ABI Prism 310. Although 13\% represents a very high level of variation for the kit, a larger samples size and number of measurement should be increased to fully test the reliability of the system. A larger sample size will also reveal with more certainty the level of allele dropout that can be encountered with the new LSB multiple. Currently there is only one locus that seems to have problems with primer binding site mutations, but given that the majority of the primers are different from the original multiplex, it is possible that with a larger sample size dropout may increase.

\section{$\underline{\text { Sensitivity of the LSB multiplex system }}$}

Of particular significance to the development of the multiplex is its performance evaluation. Performance criteria included peak heights, intercolor, intralocus, and intracolor balance. With 1ng of DNA template peak heights were evaluated to verify that homozygous peaks were not off scale and the overall peak amplitude was sufficient to allow for detection of 1 ng of template or less. Intercolor balance was also evaluated in relation to the ability to produce full profiles within the recommended range. Although intralocus balance greater than $70 \%$ was not achieved with the tested samples, the multiplex can produce full profiles under the recommended concentration range of 0.1 to 1 nanograms as the overall peak height ratio was greater than $50 \%$ for all loci 
except TPOX and their peak height were above 200RFUs. It is known that the heights of the peaks will vary from sample-to-sample, even for the same DNA sample amplified in parallel. Because the three singles source samples were not amplified at least in duplicates for any given concentration it becomes more difficult to explain if the low intralocus balance was influenced perhaps by inappropriate dilution, inhibition or pipetting error.

The range of sensitivity of this assay indicates that the LSB can produce profiler under a wide range of DNA template concentration, however is not suitable for low copy number. The current average peak heights showed no indication of saturation as the peaks were within the detection range for the ABI Prism 310, however using more than 1ng with the current multiplex will cause higher allele imbalance, incorrect allele sizing and incomplete adenylation

\section{$\underline{\text { Sizing Precision }}$}

During this study both brackets and alleles were co-electrophoresed to test their migration consistency and reproducibility on the ABI Prism 310 genetic analyzer system. The principle is to expose both known brackets and questioned alleles to the same electrophoretic conditions, assuming that each LSB bracket pair will size its respective alleles each time a sample was tested. The sample reproducibility (based on sizes generated by ROX-500) was assessed through the calculation of SDs around mean values for each bracket and its discrete alleles. The study demonstrated that on average most brackets and alleles would fall within the $0.5 \mathrm{bp}$ limit; however, for a confidence interval of $99.7 \%$, this might not be the case. JOE-labeled brackets and alleles showed more variation than the FAM-labeled ones but it is worth noticing that these samples were only run on three separate occasions, and that perhaps the number of replications would have to be larger in order to get a more accurate sizing reproducibility. 


\section{Genotype consistencies}

One key aspect of any genotyping system is the ability to provide reproducible results. The LSB multiplex was able to demonstrate in all three injections that the sample sizes and genotypes were reproducible, although in various loci the results were not accurate. The genotype inconsistencies however can be justified as the sizes for these alleles were also inaccurately sized by R0X-500.

\section{$\underline{\text { LSB kit limitations }}$}

Although the LSB multiplex can be used to amplify non-degraded samples and produce full genetic profiles the kit still remains with limitations that can potentially challenge the reliability of the system. These limitations are described below:

\section{$\underline{\text { Allele dropout }}$}

The issue of allele dropout in loci vWA and D13S317 that contributed to the failure of the previous validation study, was successfully overcome in the new LSB multiplex by selecting primers free of point mutations as well as primers with reduced potential competition for building blocks, and undesired complementarity both between primers (primer dimer) and primers and genomic DNA template (mispriming). However, the newly discover point mutation at locus TPOX during rigorous preliminary research creates future problems of heterozygous allele imbalance as observed during the peak height ratio and sensitivity study, as well as unreliable results given that heterozygous alleles may appear as homozygous specially in templates with low DNA concentrations. 


\section{$\underline{\text { Size overlap }}$}

A multiplex with same-dye labeled alleles with potential for overlap with neighboring loci and in this case overlap with a locus specific bracket, creates problems for data analysis and interpretation increasing the potential for improper genotyping of a sample alleles. Although the overlap at loci CSF1PO \& D18S1358 was not observed in the sample included in this project, it is expected to happen with samples that carry the smallest alleles for both of these loci.

\section{Need for new genotyping software}

The Oligocode genotyping software is absolutely necessary to genotype large data sets with the LSB method. The software has been specifically developed to analyze data that does not contain the internal size standard or an allelic ladder, but simply the sample alleles and the brackets.

Although the Oligocode manual claims to uses the local southern method for genotyping unknown alleles, it is unclear how the software manages to use the local southern method with only two data points (both short and long brackets) present in the data set when local southern requires at least 4 data points to create a standard curve. The original LSB work [36] does mention that analysis can be done with only the LSBs as internal lane standards or the LSBs plus two known alleles for each locus as the external lane standard; however, it does not explain how this apply to every new data set.

Currently the software only works with brackets that are equivalent in size to a full repeat but not a partial one. This makes the new LSB impossible to genotype with the software as few of the brackets synthesized to genotype the current LSB multiplex, contain partial repeats. 


\section{Incomplete bracket set.}

In order to genotype all 13 loci using the LSB method, all brackets need to be present. At this point there are only 14 brackets that can calibrate 7 loci.

\section{$\underline{\text { Future research }}$}

The new human LSB multiplex remains with issues that make it unsuitable for forensic DNA analysis. In order to overcome this problem a new primer must be designed to solve for TPOX allele dropout. New primers must also be designed for loci D18S51 and CSF1PO to overcome the size overlap problems. If good primer candidates cannot be found for these loci, mobility modifiers or spacers can be implemented in order to keep the current primers. New TAMRA \& ROX-labeled brackets must be designed to complete the set needed to calibrate all 13 loci. Oligocode software must be improved so that it can accommodate these new allele and LSB sizes. Once these issues have been solved, a new validation study must be done with a larger samples size.

Currently new STR technology and instruments are constantly being developed. Part of the technology includes kits with additional markers. Given that the current kit only amplifies all 13 loci, perhaps new markers can be included within the future-improved version of the LSB multiplex, making the kit more marketable. A new improved version of the kit may also encourage the development of commercial software for genotyping STR data without the use of an internal size standard or an allelic ladder and instead recognizes the presence of brackets. Given the current standards and regulations for validating a kit to be use in casework samples, the LSB kit might not be the system of choice; however, everyday many non-criminal paternity cases are being processed in different labs and a kit like the LSBs may become useful as the kit 
in itself might be cheaper to process, reagents costs may decrease, and sample throughput may increase.

Having a kit like locus-specific bracket may benefit the forensic community as kits available from Applied Biosystems, or Promega are highly expensive and in many ways limited to their respective software and genetic analyzers. If an improved version of the LSB kit can be in the future created, one free of allelic dropout and duly validated, this kit may become valuable to many labs searching for cheaper and better kits. 


\section{References}

[1] J. Butler, in Fundamental of Forensic DNA typing, Academic Press, 2010, pp. 205-220.

[2] L. ES, "International Human Genome Sequencing Consortium. Initial sequencing and analysis of human genome.," Nature, vol. 409, pp. 860-921, 2001.

[3] B. C. R. Budowle, "Source Attribution of a Forensic DNA Profile," Forensic Science Communications, vol. 2, no. 3, 2000.

[4] C. H. B. John M. Butler, "Improved Analysis of Short Tandem Repeats With Time-of-Flight Mass Spectromety," U.S. Department of Justice , 2001.

[5] C. I. S. Trevor J Pemberton, "Sequence determinants of human microsatellite variability," BMC Genomics, vol. 10, no. 612, 2009.

[6] J. M. Butler, "Fundamentals of Forensic DNA typing," Academic Press, 2010, pp. 147-170.

[7] J. C. H. Allison P. Null, "Genotyping of Simple and Compound Short Tandem Repeat Loci Using Electrospray Ionization Fourier Transform Ion Cyclotron Resonance Mass Spectrometry," Analytical Chemistry, vol. 73, pp. 4514-4521, 2001.

[8] N. James, "Federeation of American Scientists," 7 December 2011. [Online]. Available: http://www.fas.org/sgp/crs/misc/R41800.pdf. [Accessed 9 February 2012].

[9] J. M. Butler, Fundamentals of Forensic DNA Typing, Academic Press, 2010, p. 259.

[10] J. Wallin, "Constructing Universal Multiplex PCR Systems for Comparative Genotyping," J Forensic Sci, vol. 47, no. 1, pp. 52-65, 2002.

[11] J. Robertson, " Evaluation of native and denaturing polyacrylamide gel electrophoresis for short tandem repeat analysis," Adv. Forensic Haemogenet, vol. 5, pp. 320-322, 1994. 
[12] E. Mansfield, "Alternative labeling techniques for automated fluorescence based analysis of PCR products," BioTechniques, vol. 15, pp. 274-279, 1993.

[13] J. M. Butler, "Forensic DNA typing by capillary electrophoresis using the ABI Prism 310 and 3100 genetic analyzers for STR analysis," Electrophoresis, vol. 10, no. 11, pp. 1397-412, 2004.

[14] B. Leclair, "Precision and Accuracy in fluorescent short tandem repeat DNA Typing: assesments of benefits imparted by the use of allelic ladders with the AmpFISTR ${ }^{\circledR}$ Profiler Plus ${ }^{\mathrm{TM}}$ kit," Electrophoresis, vol. 25, pp. 790-796, 2004.

[15] J. W. Schumm, "Why Use a Size Marker and Allelic Ladders in STR Analysis?," GENEPRINTTM PRODUCTS, 1997.

[16] J. Butler, "Short Tandem Repeat Analysis for Human Identity Testing (Current Protocols in Human Genetics)," National Institute of Standards and Technology, Gaithersburg .

[17] J. K. S. E. M. Elder, "Measurement of DNA length by gel electrophoresis II: Comparison of methods for relating mobility to fragment length," Analytical Biochemistry, vol. 128, pp. 227-231, 1983.

[18] S. P. Walsh and N. J. Fildes, "Sequence analysis and characterization of stutter products at the tetranucleotide repeat locus vWA," Nucleic Acids Research, vol. 24, no. 14, p. 807-2812, 1996.

[19] B. Leclair, "Systematic Analysis of Stutter Percentage and Allele Peak Height and Peak Area Ratios at Heterozygous STR Loci for Forensic Casework and Database samples," J Forensic Sci, vol. 49, no. 5, pp. 1-13, 2004.

[20] B. Leclair, "Modulation of non-templated nucleotide addition by Taq DNA polymerase: primer modifications that facilitate genotyping," Biotechniques, vol. 20, pp. 1004-1010, 1996.

[21] J. Butler, "The Development of Reduced Size STR Amplicons as Tools for Analysis of Degraded DNA," Journal of Forensic Science, vol. 48, no. 5, 2003. 
[22] D. W. Wang, "Applied Biosystems," 2008. [Online]. Available: http://www3.appliedbiosystems.com/cms/groups/applied_markets_marketing/documents/gener aldocuments/cms_053733.pdf. [Accessed 9 February 2012].

[23] R. L. M. Huel, "Variant Alleles, Triallelic Patterns, and Point Mutations Observed in Nuclear Short Tandem Repeat Typing of Populations in Bosnia and Serbia," Croat Med J., vol. 48, pp. 494-502, 2007.

[24] D. R. Paoletti, "Empirical Analysis of the STR Profiles Resulting from Conceptual Mixtures," Journal of Forensic Science, vol. 50, no. 6, pp. 1-6, 2005.

[25] B. E. Krenke, "Validation of a 16-Locus Fluorescent Multiplex System," Journal of Forensic Science, vol. 47, no. 4, pp. 1-13, 2002.

[26] J. Butler, "STRbase," [Online]. Available: http://www.cstl.nist.gov/strbase/. [Accessed 10 January 2011].

[27] M. Edwards, "Multiplex PCR: Advantages, development, and applications," Genome Res, vol. 3, pp. S65-S75, 1994.

[28] D. T. Nock and B. R. Mc Cord, "Temperature and pH studies of short tandem repeat systems using capillary electrophoresis at elevated pH," Electrophoresis, vol. 22, p. 755-62, 2001.

[29] B. McCord, "Troubleshooting Capillary Electrophoresis Systems," Promega, 2003.

[30] J. Fadlovich, "Applera Corporation: The emerging Molecular Diagnostics Industry," Kellogg School of Management, 2003.

[31] S. Fordyce L, "High-throughput sequencing of the STR loci for forensic genetic investigation using the Roche genome sequencer FLX platform," Biotechniques, vol. 57, pp. 127-132, 2011.

[32] S. A. Greensppon, "Microchip Capillary Electrophoresis: Progress Toward an Integrated Forensic 
Analysis System".

[33] P. Dau, "DNA bracketing locus compatible standards for electrophoresis," U.S. Patent 6,013,444. .

[34] T. Serensits, "SoftGenetics," [Online]. Available:

http://www.softgenetics.com/LocusSpecificBracketSTRAnalysis.pdf. [Accessed 24 January 2011].

[35] J. Butler, "Locus-Specific Brackets for reliable typing of Y-chromosome short tandem repeats markers," Electrophoresis, vol. 26, pp. 2583-2590, 2005.

[36] P. Dau, " Multiplex Amplification and Analysis of Selected STR," LSB US patent 0186727A1, 2003.

[37] J. M. Butler, "Validation Overview," National Institute of Standards and Technology (NIST), 2005.

[38] K. WJ, " BLAT - the BLAST-like alignment tool.," Genome Res, vol. 4, pp. 656-64, 2002.

[39] "Life Technologies," Applied Biosystems, 2002. [Online]. Available: http://www3.appliedbiosystems.com/cms/groups/mcb_support/documents/generaldocuments/c ms_042511.pdf. [Accessed 1404 2012].

[40] C. J. Word, "Peak Height Ratios," in 21st International Symposium on Human Identification Mixture Interpretation Workshop, San Atonio TX, 2010.

[41] J. Butler, Forensic DNA Typing: Biology and Technology Behind STR Markers, San Diego: Elsevier Academic Press, 2001.

[42] R. D. Chrambach A, "Polyacrylamide gel electrophoresis," Science, pp. 440-51, 1971.

[43] J. Jacob V. Maizel, "SDS polyacrylamide gel electrophoresis," Trends in Biochemical Sciences, vol. 25 , no. 12 , p. 590-592, 2000.

[44] "National Diagnostics," [Online]. Available: http://www.nationaldiagnostics.com/article_info.php/articles_id/51. [Accessed 28 February 2012]. 
[45] "Methods in Biotechnology," 20 September 2009. [Online]. Available: http://biotechmethods.blogspot.com/2009/09/polyacrylamide-gel-electrophoresis.html. [Accessed 28 February 2012].

[46] R. Trachan, Human Molecular Genetics. 2nd edition., New York: Wiley-Liss, 1999.

[47] P. Gwynne and G. Heebner, "PCR AND CLONING: A TECHNOLOGY for the 21st CENTURY," Science, 9 February 2001. [Online]. Available: http://www.sciencemag.org/site/products/pcr.xhtml. [Accessed 28 February 2012].

[48] C. P. Davis, Molecular Biology: Understanding the genetic revolution, Elsevier, 2011.

[49] Invitrogen, "Topo TA Cloning User Manual," Invitrogen, 2006.

[50] L. Young, "Two-step Total Gene Synthesis Method," Nucleic Acids Reseach, vol. 32, no. 7, pp. 1-6, 2004.

[51] J. M. Butler, Fundamentals of Forensic DNA Typing, Academic Press, 2010.

[52] P. De Bievre, "The Fitness for Purpose of Analytical Methods: A Laboratory Guide to Method Validation and Related Topics," EURACHEM Working Group.

[53] J. Butler, "Validation workshop HID University Future Trends in Forensic DNA Technology," 2006.

[54] A. Carracedo, DNA typing protocols. Constructing STR Multiplex Assays., vol. 292, Human Press, 2005, pp. 53-65.

[55] SWGDAM, "SWGDAM Interpretation Guideline for Autosomal STR Typing by Forensic DNA Testing Laboratories," [Online]. Available: http://w’ww.fbi.gov/about-us/lab/codis/swgdaminterpretation-guidelines. [Accessed 24 November 2010].

[56] P. J. Collins, "Developmental validation of a single -tube amplification of the 13 CODIS STR loci, D2S1358, D19S433, and amelogenin: the Amplstar identifiler PCR amplification kit," J Forensic Sci, 
vol. 49, no. 6, pp. 1-12, 2004. 
$\underline{\text { Appendix }}$ 
Table 3. Original LSB primer sequences used to amplify the multiplex used during the validation study and current primer sequences according to the LSB manual.

Name

Current primer sequences

Original primer sequences 5' - 3'

5'- 3'

D13S317.F ACT CTGACCCATCTA

ACGCCT A

D13S317R CAG CCC AAA AAG ACA GAC AGA AAG

TH01.F CAA AGG GTA TCT GGG CTC $\mathrm{T}$

TH01.R TGG GCT GAA AAG CTC CCG ATT A

TPOX.F TGG CAC AGA ACA GGC ACT TAG

TPOX.R GAA CTG GGA ACC ACA CAG $\mathrm{G}$

Amel.F CCT GGG CTC TGT AAA GAA TAG TG

Amel.R ATC AGA GCT TAA ACT GGG AAG CTG

D5S818.F GAC AAG GGT GAT TTT CCT CTT TGG T

D5S818.R CAA GTG ATT CCA ATC ATA GCC ACA G

D7S820.F TCA GGC TGA CTA TGG AGT TAT TTT AAG G

D7S820.R CAT TTA TCC TCA TTG ACA GAA TTG CAC C

FGA.F ATG CCC CAT AGG TTT TGA ACT CAC

FGA.R TCT CAG ATC CTC TGA CAC TCG

vWA.F GAG ATG TGA AAG CCC TAG TGG A

vWA.R AAA TAC ATA GGA TGG ATG GAT AGA TGG A

D21S11.F AAT TCC CCA AGT GAA TTG CCT TCT A

D21S11.R TAT TAG TCA ATG TTC TCC AGA GAC AGA C

D18S51.F TTC ATG CCA CTG CAC TTC ACT CT

D18S51.R CCG ACT ACC AGC AAC AAC $\mathrm{AC}$
TCTGACCCATCTAACGCCTAT

GCCCAAAAAGACAGACAGAAAGA

GGCAAATAGGGGGCAAAATTCAAAG

GAAAAGCTCCCGATTATCCAG

CTTCCTCTGCTTCACTTTTCACC

CCTTCTGTCCTTGTCAGCGTTTA

CCTGGGCTCTGTAAAGAATAGTG

ATCAGAGCTTAAACTGGGAAGCTG

GACAAGGGTGATTTTCCTCTTTGGT

GTGATTCCAATCATAGCCACAG

AGGCTGACTATGGAGTTATTTTAAGG

TTATCCTCATTGACAGAATTGCAC

GCAGGGCATAACATTATCCAAAAG

GATCCTCTGACACTCGGTTGTA

GATGTGAAAGCCCTAGTGGA

CATAGGATGGATGGATAGATGGA

AATTCCCCAAGTGAATTGCCTTCTA

TCAATGTTCTCCAGAGACAGAC

TTCATGCCACTGCACTTCACTCT

CCGACTACCAGCAACAACAC 


\begin{tabular}{|c|c|c|}
\hline D3S1358.F & $\begin{array}{l}\text { TGC AGT CCA ATC TGG GTG } \\
\text { ACA GA }\end{array}$ & ACTGCAGTCCAATCTGGGT \\
\hline D3S1358.R & $\begin{array}{l}\text { TGA AAT CAA CAG AGG CTT } \\
\text { GCA TGT }\end{array}$ & ATGAAATCAACAGAGGCTTG \\
\hline D8S1179.F & $\begin{array}{l}\text { ATG TAT TTT TGT ATT TCA } \\
\text { TGT GTA CAT TCG }\end{array}$ & ATGTATTTTTGTATTTCATGTGTACATTCG \\
\hline D8S1179.R & $\begin{array}{l}\text { CAC GTA GCT ATA ATT AGT } \\
\text { TCA TTT TCA TCA }\end{array}$ & CACGTAGCTATAATTAGTTCATTTTCATCA \\
\hline D16S539.F & $\begin{array}{l}\text { TGT ACA AGT GCC AGA TGC } \\
\text { TCG TT }\end{array}$ & TGTACAAGTGCCAGATGCTCGTT \\
\hline D16S539.R & $\begin{array}{l}\text { CCA TTT ACG TTT GTG TGT } \\
\text { GCA TCT GTA A }\end{array}$ & CCATTTAGCGTTTGTGTGTGCATCTGT \\
\hline CSF1PO.F & $\begin{array}{l}\text { TGA GTC TGC CAA GGA CTA } \\
\text { GC }\end{array}$ & CTGTGTCTCAGTTTTCCTACCTGT \\
\hline CSF1PO.R & $\begin{array}{l}\text { CAC ACA CCA CTG GCC ATC } \\
\text { TTC }\end{array}$ & TGGAGGTCATCCTTATCTCCTTTC \\
\hline
\end{tabular}


Table 4 Primer concentrations for multiplex optimization (same dye only). Initial concretion were provided by previous researcher and the optimize concentrations were needed to obtained an improved version.

\begin{tabular}{|c|c|c|}
\hline Locus & Initial concentration & Optmized Concentration \\
\hline \multicolumn{3}{|l|}{ D13S317 } \\
\hline Forward & $3.8 \mathrm{pmol} / \mu 1$ & $3.9 \mathrm{pmol} / \mu \mathrm{l}$ \\
\hline Reverse & $3.8 \mathrm{pmol} / \mu \mathrm{l}$ & $3.9 \mathrm{pmol} / \mu \mathrm{l}$ \\
\hline \multicolumn{3}{|l|}{ TH01 } \\
\hline Forward & $6.8 \mathrm{pmol} / \mu \mathrm{l}$ & $6.0 \mathrm{pmol} / \mu \mathrm{l}$ \\
\hline Reverse & $6.8 \mathrm{pmol} / \mu \mathrm{l}$ & $6.0 \mathrm{pmol} / \mu \mathrm{l}$ \\
\hline \multicolumn{3}{|l|}{ TPOX } \\
\hline Forward & $6.7 \mathrm{pmol} / \mu 1$ & $6.7 \mathrm{pmol} / \mu \mathrm{l}$ \\
\hline Reverse & $6.7 \mathrm{pmol} / \mu \mathrm{l}$ & $6.7 \mathrm{pmol} / \mu \mathrm{l}$ \\
\hline \multicolumn{3}{|c|}{ Amelogenin } \\
\hline Forward & $1.6 \mathrm{pmol} / \mu \mathrm{l}$ & $1.0 \mathrm{pmol} / \mu \mathrm{l}$ \\
\hline Reverse & $1.6 \mathrm{pmol} / \mu \mathrm{l}$ & $1.0 \mathrm{pmol} / \mu \mathrm{l}$ \\
\hline \multicolumn{3}{|l|}{ D5S818 } \\
\hline Forward & $1.2 \mathrm{pmol}$ & $1.0 \mathrm{pmol} / \mu \mathrm{l}$ \\
\hline Reverse & $1.2 \mathrm{pmol}$ & $1.0 \mathrm{pmol} / \mu \mathrm{l}$ \\
\hline \multicolumn{3}{|l|}{ D7S820 } \\
\hline Forward & $12.9 \mathrm{pmol} / \mu \mathrm{l}$ & $11.5 \mathrm{pmol} / \mu \mathrm{l}$ \\
\hline Reverse & $12.9 \mathrm{pmol} / \mu \mathrm{l}$ & $11.5 \mathrm{pmol} / \mu \mathrm{l}$ \\
\hline \multicolumn{3}{|l|}{ FGA } \\
\hline Forward & $6.7 \mathrm{pmol} / \mu \mathrm{l}$ & $6.3 \mathrm{pmol} / \mu \mathrm{l}$ \\
\hline $\begin{array}{l}\text { Reverse } \\
\text { vWA }\end{array}$ & $6.7 \mathrm{pmol} / \mu 1$ & $6.3 \mathrm{pmol} / \mu \mathrm{l}$ \\
\hline \multicolumn{3}{|l|}{ vWA } \\
\hline Forward & $9.2 \mathrm{pmol} / \mu \mathrm{l}$ & $8.5 \mathrm{pmol} / \mu \mathrm{l}$ \\
\hline Reverse & $9.2 \mathrm{pmol} / \mu \mathrm{l}$ & $8.5 \mathrm{pmol} / \mu \mathrm{l}$ \\
\hline \multicolumn{3}{|l|}{$\mathrm{D} 21 \mathrm{~S} 11$} \\
\hline Forward & $14.2 \mathrm{pmol} / \mu \mathrm{l}$ & $15.3 \mathrm{pmol} / \mu \mathrm{l}$ \\
\hline Reverse & $14.2 \mathrm{pmol} / \mu \mathrm{l}$ & $15.3 \mathrm{pmol} / \mu \mathrm{l}$ \\
\hline \multicolumn{3}{|l|}{ D18S51 } \\
\hline Forward & $15.7 \mathrm{pmol} / \mu \mathrm{l}$ & $13.0 \mathrm{pmol} / \mu \mathrm{l}$ \\
\hline Reverse & $15.7 \mathrm{pmol} / \mu \mathrm{l}$ & $13.0 \mathrm{pmol} / \mu \mathrm{l}$ \\
\hline \multicolumn{3}{|l|}{ D3S1358 } \\
\hline Forward & $5.8 \mathrm{pmol} / \mu \mathrm{l}$ & $5.5 \mathrm{pmol} / \mu \mathrm{l}$ \\
\hline Reverse & $5.8 \mathrm{pmol} / \mu \mathrm{l}$ & $5.5 \mathrm{pmol} / \mu \mathrm{l}$ \\
\hline \multicolumn{3}{|l|}{ D8S1179 } \\
\hline Forward & $14.5 \mathrm{pmol} / \mu \mathrm{l}$ & $15.1 \mathrm{pmol} / \mu \mathrm{l}$ \\
\hline Reverse & $14.5 \mathrm{pmol} / \mu \mathrm{l}$ & $15.1 \mathrm{pmol} / \mu \mathrm{l}$ \\
\hline \multicolumn{3}{|l|}{ D16S539 } \\
\hline Forward & $2.6 \mathrm{pmol} / \mu \mathrm{l}$ & $2.9 \mathrm{pmol} / \mu \mathrm{l}$ \\
\hline Reverse & $2.6 \mathrm{pmol} / \mu \mathrm{l}$ & $2.9 \mathrm{pmol} / \mu \mathrm{l}$ \\
\hline \multicolumn{3}{|l|}{ CSF1PO } \\
\hline Forward & $10.3 \mathrm{pmol} / \mu \mathrm{l}$ & $9.5 \mathrm{pmol} / \mu \mathrm{l}$ \\
\hline Reverse & $10.3 \mathrm{pmol} / \mu \mathrm{l}$ & $9.5 \mathrm{pmol} / \mu \mathrm{l}$ \\
\hline
\end{tabular}


Table 5. Optimized primer concentration for LSB multiplex (all colors)

\begin{tabular}{|c|c|}
\hline Name & Optimized Concentration \\
\hline \multicolumn{2}{|l|}{ D13S317 } \\
\hline Forward & $1.0 \mathrm{pmol} / \mu \mathrm{l}$ \\
\hline Forward. FDT & $1.0 \mathrm{pmol} / \mu \mathrm{l}$ \\
\hline Reverse & $2.0 \mathrm{pmol} / \mu \mathrm{l}$ \\
\hline \multicolumn{2}{|l|}{ TH01 } \\
\hline Forward & $26.0 \mathrm{pmol} / \mu \mathrm{l}$ \\
\hline Reverse & $26.0 \mathrm{pmol} / \mu \mathrm{l}$ \\
\hline \multicolumn{2}{|l|}{ TPOX } \\
\hline Forward & $27.6 \mathrm{pmol} / \mu \mathrm{l}$ \\
\hline Reverse & $26.6 \mathrm{pmol} / \mu \mathrm{l}$ \\
\hline \multicolumn{2}{|l|}{ Amelogenin } \\
\hline Forward & $1.4 \mathrm{pmol} / \mu \mathrm{l}$ \\
\hline Reverse & $1.4 \mathrm{pmol} / \mu \mathrm{l}$ \\
\hline \multicolumn{2}{|l|}{ D5S818 } \\
\hline Forward & $5.0 \mathrm{pmol} / \mu \mathrm{l}$ \\
\hline Reverse & $5.6 \mathrm{pmol} / \mu \mathrm{l}$ \\
\hline \multicolumn{2}{|l|}{ D7S820 } \\
\hline Forward & $22.7 \mathrm{pmol} / \mu \mathrm{l}$ \\
\hline Reverse & $23.8 \mathrm{pmol} / \mu \mathrm{l}$ \\
\hline \multicolumn{2}{|l|}{ FGA } \\
\hline Forward & $25.0 \mathrm{pmol} / \mu \mathrm{l}$ \\
\hline Reverse & $25.0 \mathrm{pmol} / \mu \mathrm{l}$ \\
\hline \multicolumn{2}{|l|}{ vWA } \\
\hline Forward & $5.0 \mathrm{pmol} / \mu \mathrm{l}$ \\
\hline Reverse & $5.0 \mathrm{pmol} / \mu \mathrm{l}$ \\
\hline \multicolumn{2}{|l|}{ D21S11 } \\
\hline Forward & $6.0 \mathrm{pmol} / \mu \mathrm{l}$ \\
\hline Reverse & $6.0 \mathrm{pmol} / \mu \mathrm{l}$ \\
\hline \multicolumn{2}{|l|}{ D18S51 } \\
\hline Forward & $25.0 \mathrm{pmol} / \mu \mathrm{l}$ \\
\hline Reverse & $25.0 \mathrm{pmol} / \mu \mathrm{l}$ \\
\hline \multicolumn{2}{|l|}{ D3S1358 } \\
\hline Forward & $8.6 \mathrm{pmol} / \mu \mathrm{l}$ \\
\hline Reverse & $7.7 \mathrm{pmol} / \mu \mathrm{l}$ \\
\hline \multicolumn{2}{|l|}{ D8S1179 } \\
\hline Forward & $50.0 \mathrm{pmol} / \mu \mathrm{l}$ \\
\hline Reverse & $50.0 \mathrm{pmol} / \mu \mathrm{l}$ \\
\hline \multicolumn{2}{|l|}{ D16S539 } \\
\hline Forward & $8.5 \mathrm{pmol} / \mu \mathrm{l}$ \\
\hline Reverse & $8.5 \mathrm{pmol} / \mu \mathrm{l}$ \\
\hline \multicolumn{2}{|l|}{ CSF1PO } \\
\hline Forward & $24.7 \mathrm{pmol} / \mu \mathrm{l}$ \\
\hline Reverse & $24.1 \mathrm{pmol} / \mu \mathrm{l}$ \\
\hline
\end{tabular}


Table 6 Template source used for cloning locus-specific brackets

\begin{tabular}{|c|c|c|c|c|}
\hline Locus & $\begin{array}{l}\text { Allele } \\
\text { Range }\end{array}$ & Sample & Bracket & $\begin{array}{l}\text { Locus Type and Number of } \\
\text { Repeats }\end{array}$ \\
\hline \multirow[t]{2}{*}{ D13S317 } & $6-13$ & BANS & Short & Homozygous locus with 8R \\
\hline & & B511 \& S511 & Long & Heterozygous locus with $10 \mathrm{R} \& 14 \mathrm{R}$ \\
\hline \multirow[t]{2}{*}{ TH01 } & 4-11 & BANS & Short & Homozygous locus with 6R \\
\hline & & B378 \& S378 & Long & Homozygous locus with 9R \\
\hline TPOX & $6-13$ & LMSF & $\begin{array}{l}\text { Short \& } \\
\text { Long }\end{array}$ & Heterozygous locus with $6 \mathrm{R} \& 12 \mathrm{R}$ \\
\hline \multirow[t]{2}{*}{ D5S818 } & $7-16$ & B999 \& S 999 & Short & Homozygous locus with 9R \\
\hline & & B188 \& S 188 & Long & Heterozygous locus with 9R \& 14R \\
\hline \multirow[t]{2}{*}{ D7S820 } & $6-15$ & B $581 \&$ S 581 & Short & Heterozygous locus with $7 \mathrm{R} \& 12 \mathrm{R}$ \\
\hline & & B551 \& S 551 & Long & Heterozygous locus with $10 \mathrm{R} \& 14 \mathrm{R}$ \\
\hline \multirow[t]{2}{*}{ FGA } & $16-52$ & S 311 & Short & Heterozygous locus with $16 R \& 19 R$ \\
\hline & & LMSF & Long & Heterozygous locus with $27 R \& 30 R$ \\
\hline \multirow[t]{2}{*}{ vWA } & $11-20$ & B114 \& S 114 & Long & Heterozygous locus with $17 R \& 20 R$ \\
\hline & & B540 \& S 540 & Short & Homozygous locus with $14 \mathrm{R}$ \\
\hline \multirow[t]{2}{*}{ D21S11 } & $24-38$ & B401 \&S 401 & Short & Heterozygous locus with $25 R \& 29 R$ \\
\hline & & B909 \& S 909 & Long & $\begin{array}{l}\text { Heterozygous locus with } 30 \mathrm{R} \& \\
34.2 \mathrm{R}\end{array}$ \\
\hline \multirow[t]{2}{*}{ D18S51 } & $9-27$ & B669 \& S 669 & Short & Heterozygous locus with $10 \mathrm{R} \& 15 \mathrm{R}$ \\
\hline & & LMSF & Long & Heterozygous locus with $15 R \& 21 R$ \\
\hline \multirow[t]{4}{*}{ D3S1358 } & $12-19$ & B007 \& S007 & Long & Heterozygous locus with $17 \mathrm{R} \& 18 \mathrm{R}$ \\
\hline & & DNA & Short & Homozygous locus with 10R \\
\hline & & Technologies & & \\
\hline & & B126 \& S 126 & Long & Heterozygous locus with $14 \mathrm{R} \& 18 \mathrm{R}$ \\
\hline \multirow[t]{3}{*}{ D8S1179 } & $7-19$ & BANS & Long & Heterozygous locus with $18 \mathrm{R} \& 19 \mathrm{R}$ \\
\hline & & B999 \& S999 & Short & Heterozygous locus with 8R \& 15R \\
\hline & & B276 \& S 276 & Short & Heterozygous locus with 8R \& 14R \\
\hline \multirow[t]{2}{*}{ D16S539 } & $5-15$ & B142 \& S 142 & Short & Heterozygous locus with 8R \& 11R \\
\hline & & $\mathrm{S} 483-\mathrm{C}$ & Long & Heterozygous locus with $12 \mathrm{R} \& 14 \mathrm{R}$ \\
\hline \multirow[t]{2}{*}{ CSF1PO } & $6-15$ & B311 \& S 311 & Short & Heterozygous locus with 7R \& 11R \\
\hline & & B972 \& S 972 & Long & Heterozygous locus with $11 \mathrm{R} \& 14 \mathrm{R}$ \\
\hline
\end{tabular}


Table 7. Primers designed for cloning locus-specific brackets. SB stands for short bracket, LB for long bracket and CSP stands for cloning specific primers.

\begin{tabular}{|c|c|c|c|}
\hline Locus & Primer sequence 5' - 3' & $\begin{array}{l}\text { Length } \\
\text { (nt) }\end{array}$ & $\begin{array}{c}\mathbf{T m} \\
\left({ }^{\circ} \mathbf{C}\right) \\
\end{array}$ \\
\hline \multicolumn{4}{|l|}{ D13S317 } \\
\hline Forward-SB & TCG CCG CCT ATC TGT ATT TAC AAA T & 25 & 56.4 \\
\hline Forward-LB & GTG ACT CTC TGG ACT CTG ACC C & 22 & 58.5 \\
\hline Forward-CSP & TCC AAG CTC ACA GTG CCT AA & 20 & 59.6 \\
\hline $\begin{array}{l}\text { Reverse-CSP } \\
\text { TH01 }\end{array}$ & TCC GAG GTT CTC TTC CTG TG & 20 & 60.4 \\
\hline Reverse-SB & CTC CCG ATT ATC CAG CCT GG & 20 & 57.3 \\
\hline Reverse-LB & CTC CCG ATT ATC CAG CCT GG & 22 & 59.4 \\
\hline Forward-CSP & CCT GGT GTT TGA GTC CCT GT & 20 & 60.0 \\
\hline Reverse-CSP & CAG CTT CAT CCT GAG CTT CC & 20 & 61.1 \\
\hline \multicolumn{4}{|l|}{ TPOX } \\
\hline Reverse-SB & TGG GAA CCC CAC AGG TTA A & 19 & 56.1 \\
\hline Reverse-LB & CAG GGG AGG AAC TGG GAA C & 19 & 57.6 \\
\hline Forward-CSP & ATG CCC AGC ACA TGC CTA & 18 & 61.2 \\
\hline Reverse-CSP & GTG TGC TCA CAC CAA GCA CT & 20 & 59.9 \\
\hline \multicolumn{4}{|l|}{${ }^{1}$ Amelogenin } \\
\hline Reverse-SB & CCT GGG CTC TGT AAA GAA TAG TG & 23 & 55.0 \\
\hline Forward-CSP & TGT GTG CTG GTT TCT GCT TC & 20 & 55.9 \\
\hline Reverse-CSP & CGG CCA TAT TTA GGA GGA CA & 20 & 54.1 \\
\hline \multicolumn{4}{|l|}{ D5S818 } \\
\hline Reverse-SB & TAG CCA CAG TTT ACA ACA TTT GTA TC & 26 & 53.7 \\
\hline Reverse-LB & GTG CTT TTT AGC CAA GTG ATT CCA & 24 & 56.1 \\
\hline Forward-CSP & ACG CCT TTC CTC TGA AGT GA & 20 & 59.9 \\
\hline Reverse-CSP & ACC TGA AAG GGA CAG GGT TT & 20 & 59.8 \\
\hline \multicolumn{4}{|l|}{ D7S820 } \\
\hline Reverse-SB & G CCA TTG ACA GAA TTG CAC CAA ATA T & 26 & 56.6 \\
\hline Reverse-LB & CGA TTC CAC ATT TAT CCT CAT TGA CAG A & 28 & 56.8 \\
\hline Forward-CSP & CCT GAC CCC CTA TGG AAT TT & 20 & 60.1 \\
\hline Reverse-CSP & TTA CAC GAT GGA AGG CAT CA & 20 & 60.7 \\
\hline \multicolumn{4}{|l|}{ FGA } \\
\hline Reverse-SB & AGA TCC TCT GAC ACT CGG TTG & 21 & 56.2 \\
\hline Reverse-LB & TTC AGG ACT TCA ATT CTG CT & 20 & 51.6 \\
\hline Forward-CSP & AGC TGG CAA GTG AGT GAT CC & 20 & 60.4 \\
\hline Reverse-CSP & GGG ACC ACA GCC ACA TAC TT & 20 & 59.9 \\
\hline
\end{tabular}




\begin{tabular}{|c|c|c|c|}
\hline vWA & & & \\
\hline Reverse-SB & TAG GAT GGA TAG ATG GAT AGA TAG ATA G & 28 & 52.3 \\
\hline Reverse-LB & TAG GAC AGA TGA TAA ATA CAT AGG ATG G & 28 & 53.8 \\
\hline Forward-CSP & TCC CAC CTT CCA GAA GAA GA & 20 & 55.2 \\
\hline Reverse-CSP & GGT GAA GTT GGG TCT GCA CT & 20 & 57.6 \\
\hline D21S11 & & & \\
\hline Reverse-SB & AAT GTT CTC CAG AGA CAG ACT & 21 & 53.0 \\
\hline Reverse-LB & CGG GGC GTG ATA TAT TAA AGA TGT TG & 26 & 55.9 \\
\hline Forward-CSP & GGT GGG CTG AGT GGA GAA TA & 20 & 59.8 \\
\hline Reverse-CSP & AGA GGC CTC ACA GGT TTT CA & 20 & 59.6 \\
\hline D18S51 & & & \\
\hline Reverse-SB & AAT GTT CTC CAG AGA CAG ACT & 21 & 53.0 \\
\hline Reverse-LB & CGG GGC GTG ATA TAT TAA AGA TGT TG & 26 & 55.9 \\
\hline Forward-CSP & AAC AAT AGG CCA AGC GTG AT & 20 & 59.60 \\
\hline Reverse-CSP & TAG CCG ACA AAA GGC TAT GG & 20 & 60.2 \\
\hline${ }^{2} \mathrm{D} 3 \mathrm{~S} 1358$ & & & \\
\hline Reverse-LB & GGC TTA TAC TCA TGA AAT CAA CAG AGG C & 28 & 57.5 \\
\hline Forward-CSP & AGC ACT TTG GAA GGC TGA GA & 20 & 60.1 \\
\hline Reverse-CSP & CCC ATA CCC TGA GCC ATA GA & 20 & 59.9 \\
\hline D8S1179 & & & \\
\hline Reverse-SB & GTA TTA GTT CAT TTT CAT CAC TGT ATC GTA & 30 & 53.5 \\
\hline Reverse-LB & AGT ATA GTT TCA CGT AGC TAT AAT TAG TTC & 30 & 52.8 \\
\hline Forward-CSP & CCT TTG CCT GAG TTT TGC TC & 20 & 59.9 \\
\hline Reverse-CSP & TGA GAC CGT GTC TTG CTC TG & 20 & 60.2 \\
\hline D16S539 & & & \\
\hline Reverse-SB & CAT CTG TAA GCA TGT ATC TAT CAT CCA TCT & 30 & 56.0 \\
\hline Reverse-LB & GGC GAG AGT GAT TCC ATT TTT ATA CCA TTT A & 31 & 57.2 \\
\hline Forward-CSP & ATC TGC CCT CAT CAG TGG AC & 20 & 60.1 \\
\hline Reverse-CSP & CAG CTA TTT GGG AGG CTG AG & 20 & 59.9 \\
\hline CSF1PO & & & \\
\hline Reverse-SB & ATC CCT GCA TCT CAG ACT CT & 20 & 55.1 \\
\hline Reverse-LB & GGT CAT CCT TAT CTC CTT TCT C & 22 & 52.2 \\
\hline Forward-CSP & TTC GAG AAG GTG GCC TCT AA & 20 & 55.4 \\
\hline Reverse-CSP & TCA CAA TCT GCT GCA AGT CC & 20 & 55.5 \\
\hline
\end{tabular}

${ }^{1}$ There is no reverse primer for the long bracket of amelogenin as the short bracket of locus D5S818 is used to size the amelogenin DNA fragments

${ }^{2}$ Due to space limitation, a reverse primer for the short bracket of D3S1358 was not designed. 